\title{
Deformation openness and closedness of various classes of compact complex manifolds; examples
}

\author{
DAN POPOVICI
}

\begin{abstract}
We review the relations between compact complex manifolds carrying various types of Hermitian metrics (Kähler, balanced, or strongly Gauduchon) and those satisfying topological properties such as the $\partial \bar{\partial}$-lemma, or the degeneration at $E_{1}$ of the Frölicher spectral sequence. We also review the behaviour of these properties under holomorphic deformations. The emphasis will be placed on the notion of strongly Gauduchon (sG) manifold that we introduced recently in the study of deformation limits of projective and Moishezon manifolds. Besides its expository aspect, the paper presents new results such as exhibiting various examples of $\mathrm{sG}$ and non-sG manifolds. These are then used to review and reinterpret from this new standpoint a range of constructions already known in the literature.
\end{abstract}

Mathematics Subject Classification (2010): 32G05 (primary); 53C55, 58A14, 58A25, 14E05 (secondary).

\section{Introduction}

The main theme of this text is the interaction between metric and topological properties satisfied by compact complex manifolds.

\subsection{Metric properties: smooth metrics}

A Hermitian metric on a given compact complex manifold $X\left(\operatorname{dim}_{\mathbb{C}} X:=n\right)$ will be identified with the associated positive-definite $C^{\infty}(1,1)$-form $\omega>0$ on $X$. The metric $\omega$ is said to be:

- Kähler if $d \omega=0$;

- Balanced ${ }^{1}$ (or semi-Kähler ${ }^{2}$ or co-Kähler) if $d \omega^{n-1}=0$;

- Strongly Gauduchon ${ }^{3}$ (or $s G$ ) if $\partial \omega^{n-1}$ is $\bar{\partial}$-exact;

- Gauduchon if $\partial \omega^{n-1}$ is $\bar{\partial}$-closed or, equivalently, if $\partial \bar{\partial} \omega^{n-1}=0$.

${ }^{1}$ Terminology of [35].

2 Terminology of [19], unrelated to the balanced condition used by Donaldson, Luo and others in the context of $\operatorname{cscK}$ metrics.

${ }^{3}$ Notion introduced in [41].

Received October 18, 2011; accepted in revised form March 28, 2012. 
Although Hermitian metrics always exist, the stronger Kähler, balanced and sG metrics need not exist on an arbitrary $X$. A compact complex manifold carrying one of the first three types of metrics described above is said to be a compact Kähler, balanced on, respectively, strongly Gauduchon (or $s G$ ) manifold. By contrast, Gauduchon metrics exist on any compact complex manifold (cf. [18]). Actually, Gauduchon's main result in [18] asserts far more: there exists a Gauduchon metric (unique up to normalisation) in the conformal class of any Hermitian metric on $X$. At the level of Hermitian metrics $\omega$ on $X$, the following obvious implications hold:

$\omega$ is Kähler $\Longrightarrow \omega$ is balanced $\Longrightarrow \omega$ is $\mathrm{SG} \Longrightarrow \omega$ is Gauduchon.

In the case of complex surfaces $X$ (i.e. $n=2)$, it is obvious that $\omega$ is a Kähler metric if and only if $\omega$ is a balanced metric. An sG metric $\omega$ need not be Kähler even when $n=2$, but compact complex surfaces $X$ carrying sG metrics also carry Kähler metrics (see [41, Section 3]). Thus, at the level of compact complex manifolds, we have the following equivalences:

$X$ is a Kähler surface $\Longleftrightarrow X$ is a balanced surface $\Longleftrightarrow X$ is an sG surface.

However, if compact complex surfaces are replaced by compact complex manifolds of complex dimension $n \geq 3$, the above equivalences break down, and only strict implications from left to right hold.

\subsection{Metric properties: currents and birational transformations}

Recall that the so-called Kähler currents provide a singular (thus weaker) substitute for Kähler metrics. A $d$-closed positive $(1,1)$-current $T$ on $X$ is said to be a Kähler current $^{4}$ if it satisfies the strong positivity condition

$$
T \geq \varepsilon \omega \quad \text { on } X
$$

for some constant $\varepsilon>0$ and some Hermitian metric $\omega>0$. Kähler currents need not exist on an arbitrary $X$, but they may exist when Kähler metrics do not. Recall that $X$ is said to be a Fujiki class $-\mathcal{C}$ manifold if $X$ is bimeromorphic to a compact Kähler manifold, or equivalently, if there exists a proper holomorphic bimeromorphic map (i.e. a modification)

$$
\mu: \widetilde{X} \rightarrow X
$$

from a compact Kähler manifold $\tilde{X}$. Fujiki introduced class-C manifolds $X$ as meromorphic images of compact Kähler manifolds in [17], while Varouchas gave them the above nice characterisation in [47]. It is a result of Demailly and Paun that class $-\mathcal{C}$ manifolds are characterised by the existence of a Kähler current.

4 Terminology introduced in [27]. 
Theorem 1.1 (Demailly-Paun [15]). A compact complex manifold $X$ is of class $\mathcal{C}$ if and only if there exists a Kähler current $T$ on $X$.

Recall that a Moishezon manifold is, by definition, a compact complex manifold that is bimeromorphic to a projective manifold. Equivalently, $X$ is Moishezon if and only if there exists a modification

$$
\mu: \widetilde{X} \rightarrow X
$$

from a projective manifold $\widetilde{X}$. Thus Moishezon manifolds are to projective manifolds what class-C manifolds are to compact Kähler manifolds.

The special case of integral cohomology classes is relevant in characterisations of some of the above classes of manifolds. Recall that the De Rham 2-cohomology class $\{\omega\} \in H_{D R}^{2}(X, \mathbb{R})$ (respectively, $\{T\} \in H_{D R}^{2}(X, \mathbb{R})$ ) defined by a $C^{\infty} d$ closed real $(1,1)$-form $\omega$ (respectively, by a $d$-closed real $(1,1)$-current $T$ ) is said to be integral if it is the first Chern class of a holomorphic line bundle $L \rightarrow X$ or, equivalently, if $\omega$ (respectively, $T$ ) is the curvature form (respectively, curvature current) $\frac{i}{\pi} \Theta_{h}(L)$ of a holomorphic line bundle $(L, h) \rightarrow X$ endowed with a $C^{\infty}$ (respectively, singular) Hermitian fibre metric $h$.

There are neat characterisations of projective and Moishezon manifolds mirroring the general case of arbitrary (i.e. possibly transcendental) classes that occur on Kähler and class- $\mathcal{C}$ manifolds.

Theorem 1.2 (Kodaira's embedding theorem). A compact complex manifold $X$ is projective if and only if there exists a Kähler metric $\omega$ on $X$ whose De Rham cohomology class $\{\omega\} \in H_{D R}^{2}(X, \mathbb{R})$ is integral.

Thus projective manifolds are integral-class special cases of compact Kähler manifolds. Likewise, Moishezon manifolds are integral-class special cases of class$\mathcal{C}$ manifolds, as the following characterisation shows:

Theorem 1.3 (Ji-Shiffman [27]). A compact complex manifold $X$ is Moishezon if and only if there exists a Kähler current $T$ on $X$ whose De Rham cohomology class $\{T\} \in H_{D R}^{2}(X, \mathbb{R})$ is integral.

An interesting result of Alessandrini and Bassanelli (see [4-6]) asserts that every class- $\mathcal{C}$ manifold is balanced (i.e. carries a balanced metric). They actually accomplished rather more proving that balanced manifolds are stable under modifications:

Theorem 1.4 (Alessandrini-Bassanelli [6]). Let $\mu: \widetilde{X} \rightarrow X$ be a modification of compact complex manifolds. Then $X$ is balanced if and only if $\widetilde{X}$ is balanced.

Now if $X$ is a class- $\mathcal{C}$ manifold, then by [47] there exists a modification $\mu$ : $\widetilde{X} \rightarrow X$ where $\widetilde{X}$ is a compact Kähler manifold. Then $\widetilde{X}$ is also balanced and, by Theorem 1.4, $X$ must be balanced as well.

It is worth mentioning that the sG condition enjoys the same modification stability property. 
Theorem 1.5 (Theorem 1.3. in [43]). Let $\mu: \widetilde{X} \rightarrow X$ be a modification of compact complex manifolds. Then $X$ is strongly Gauduchon if and only if $\widetilde{X}$ is strongly Gauduchon.

Once again, the picture is known to be much simpler in the case of compact complex surfaces (i.e. when $n=2$ ): all class-C $\mathcal{C}$ surfaces are actually Kähler, while all Moishezon surfaces are actually projective. However, in dimension $n \geq 3$, there exist Moishezon manifolds that are not projective and class-C manifolds that are not Kähler (see e.g. the examples of Hironaka and Moishezon described in [21, Appendix B, Example 3.4.2.], from which the only known class of class-C manifolds that are neither Kähler nor Moishezon can be obtained). A host of other examples are provided by Lebrun's twistor spaces in [33]: these are all Moishezon (hence class-C ) 3-dimensional compact complex manifolds that are not Kähler.

\subsection{Topological properties}

First recall the following standard piece of terminology.

Definition 1.6. A compact complex manifold $X$ is said to satisfy the $\partial \bar{\partial}$-lemma if, for any $d$-closed pure-type form $u$ on $X$, the following exactness properties are equivalent:

$$
u \text { is } d \text {-exact } \Longleftrightarrow u \text { is } \partial \text {-exact } \Longleftrightarrow u \text { is } \bar{\partial} \text {-exact } \Longleftrightarrow u \text { is } \partial \bar{\partial} \text {-exact }
$$

If the pure-type assumption on $u$ is dropped, then $u$ must be assumed to be both $d$-closed and $d^{c}$-closed (or, equivalently, both $\partial$-closed and $\bar{\partial}$-closed) before the above exactness properties are required to be equivalent (cf. [14]). For a pure-type form $u$, the sole $d$-closedness is equivalent to $u$ being both $\partial$-closed and $\bar{\partial}$-closed. However, if $u$ is not of pure-type, $d u=0$ does not imply $\partial u=0$ and $\bar{\partial} u=0$.

It is a standard fact in Hodge theory that any compact Kähler manifold satisfies the $\partial \bar{\partial}$-lemma. Moreover, if $\mu: \widetilde{X} \rightarrow X$ is a modification between compact complex manifolds and if the $\partial \bar{\partial}$-lemma holds for $\widetilde{X}$, then the $\partial \bar{\partial}$-lemma also holds for $X$ (see e.g. [14, Theorem 5.22.]). In particular, class- $\mathcal{C}$ manifolds (hence also Moishezon manifolds) satisfy the $\partial \bar{\partial}$-lemma.

Now recall that on any compact complex manifold $X$, we always have

$$
b_{k}(X) \leq \sum_{p+q=k} h^{p, q}(X), \quad k=0,1, \ldots, 2 n=\operatorname{dim}_{\mathbb{R}} X,
$$

where $b_{k}(X):=\operatorname{dim}_{\mathbb{C}} H_{D R}^{k}(X, \mathbb{C})$ is the $k$-th Betti number of $X$ and $h^{p, q}(X):=$ $\operatorname{dim}_{\mathbb{C}} H^{p, q}(X, \mathbb{C})$ is the Hodge number of type $(p, q)$ of $X$. The Dolbeault cohomology group associated with $(p, q)$-forms on $X$ is denoted, as usual, by $H^{p, q}(X, \mathbb{C})$. Recall that the Frölicher spectral sequence of $X$ degenerates at $E_{1}$ (a property that will be denoted by $E_{1}(X)=E_{\infty}(X)$ ) if and only if equality is achieved in all of the above inequalities (1.1). Indeed, we always have $H_{D R}^{k}(X, \mathbb{C})=\bigoplus_{p+q=k} E_{\infty}^{p, q}(X)$ for all $k$ and $E_{1}^{p, q}(X)=H^{p, q}(X, \mathbb{C})$, as well as $\operatorname{dim} E_{\infty}^{p, q}(X) \leq \operatorname{dim} E_{1}^{p, q}(X)$, for all $p, q$. Frölicher degeneration at $E_{1}$ means that $E_{1}^{p, q}(X)=E_{\infty}^{p, q}(X)$ for all $p, q$. Hence we have: 
Proposition 1.7 (Standard). On any compact complex manifold $X\left(\operatorname{dim}_{\mathbb{C}} X=: n\right)$, the property $E_{1}(X)=E_{\infty}(X)$ is equivalent to the existence of a Hodge decomposition on $X$, possibly without Hodge symmetry, in the form:

$$
H_{D R}^{k}(X, \mathbb{C}) \simeq \bigoplus_{p+q=k} H^{p, q}(X, \mathbb{C}), \quad k=0,1, \ldots, 2 n,
$$

where the isomorphism need not be canonical.

On the other hand, recall that the $\partial \bar{\partial}$-lemma implies both the Hodge decomposition and the Hodge symmetry. In particular, the Frölicher spectral sequence of any compact complex manifold $X$ that satisfies the $\partial \bar{\partial}$-lemma degenerates at $E_{1}$.

Indeed, it is easily verified that the $\partial \bar{\partial}$-lemma implies that every Dolbeault cohomology class of type $(p, q)$ can be represented by a $d$-closed $(p, q)$-form. This allows one to define, for every $(p, q)$, a linear map $H^{p, q}(X, \mathbb{C}) \longrightarrow H_{D R}^{p+q}(X, \mathbb{C})$ by sending every Dolbeault class to the De Rham class of any of its $d$-closed representatives. This De Rham class is independent of the choice of the $d$-closed representative of the initial Dolbeault class. Furthermore, the $\partial \bar{\partial}$-lemma also implies that the map defined in this way for every $(p, q)$ is injective. Consequently, when the $\partial \bar{\partial}$-lemma holds on $X$, there is, for every $k$, a canonical injection

$$
\bigoplus_{p+q=k} H^{p, q}(X, \mathbb{C}) \hookrightarrow H_{D R}^{k}(X, \mathbb{C})
$$

which must be an isomorphism thanks to the reverse-way dimension inequality (1.1) which always holds on $X$.

The $\partial \bar{\partial}$-lemma is also known to imply the Hodge symmetry because:

- On any $X$, there are well-defined linear maps from the Bott-Chern cohomology to the Dolbeault cohomology

$$
H_{B C}^{p, q}(X, \mathbb{C}) \longrightarrow H^{p, q}(X, \mathbb{C}), \quad p, q=0,1, \ldots, n=\operatorname{dim}_{\mathbb{C}} X,
$$

mapping the Bott-Chern cohomology class $[u]_{B C}$ of any $d$-closed $(p, q)$-form $u$ to the Dolbeault cohomology class $[u]$ of the same form $u$;

- On any $X$, the map $H_{B C}^{p, q}(X, \mathbb{C}) \ni[u]_{B C} \mapsto[\bar{u}]_{B C} \in H_{B C}^{q, p}(X, \mathbb{C})$ is welldefined, $\mathbb{C}$-anti-linear and bijective, so the Hodge symmetry analogue for the BottChern cohomology always holds in a trivial way;

- If the $\partial \bar{\partial}$-lemma holds on $X$, the maps (1.2) are easily seen to be isomorphisms.

The conclusion is summed up in the following:

Proposition 1.8 (Standard). If the $\partial \bar{\partial}$-lemma holds on a compact complex manifold $X\left(\operatorname{dim}_{\mathbb{C}} X:=n\right)$, then there are canonical isomorphisms:

$$
H_{D R}^{k}(X, \mathbb{C}) \simeq \bigoplus_{p+q=k} H^{p, q}(X, \mathbb{C}) \text { and } H^{p, q}(X, \mathbb{C}) \simeq \overline{H^{q, p}(X, \mathbb{C})}
$$

for all $k=0,1, \ldots, 2 n$ and all $p, q=0,1, \ldots, n$. 
As usual, the situation is much simpler on surfaces than in higher dimension: every compact complex surface $X$ satisfies the property $E_{1}(X)=E_{\infty}(X)$ (see e.g. [8]), while the only compact complex surfaces satisfying the $\partial \bar{\partial}$-lemma are the Kähler ones. The latter statement can either be verified directly or seen using sG surfaces: the $\partial \bar{\partial}$-lemma always implies the sG property (see next subsection), while for compact complex surfaces the sG property and the Kähler property are equivalent (see Subsection 1.1).

\subsection{Metric-topological interplay}

The $\partial \bar{\partial}$-lemma property and the balanced property are unrelated (see $e . g$. examples below), but they are both implied by the class- $\mathcal{C}$ property and, in turn, they both imply the $s G$ property.

Let us recall the argument given in [41] proving that on every compact complex manifold $X$ which satisfies the $\partial \bar{\partial}$-lemma, the notions of Gauduchon and sG metrics are equivalent, hence every such $X$ is an sG manifold. If $\omega$ is a Gauduchon metric on $X$, then the pure-type $(n, n-1)$-form $\partial \omega^{n-1}$ is $\bar{\partial}$-closed by definition. Since $\partial \omega^{n-1}$ is also $\partial$-closed, it must be $d$-closed. Thus, if the $\partial \bar{\partial}$-lemma holds on $X$, the $d$-closed and $\partial$-exact pure-type form $\partial \omega^{n-1}$ must also be $\bar{\partial}$-exact. Hence $\omega$ is an sG metric on $X$.

While the sG property of $X$ and the degeneration at $E_{1}$ of the spectral sequence of $X$ are unrelated (see Theorem 1.11 below), we have seen above that they are both implied by the $\partial \bar{\partial}$-lemma.

The relations among these properties for a compact complex manifold $X$ are summed up in the following diagram:

(*)

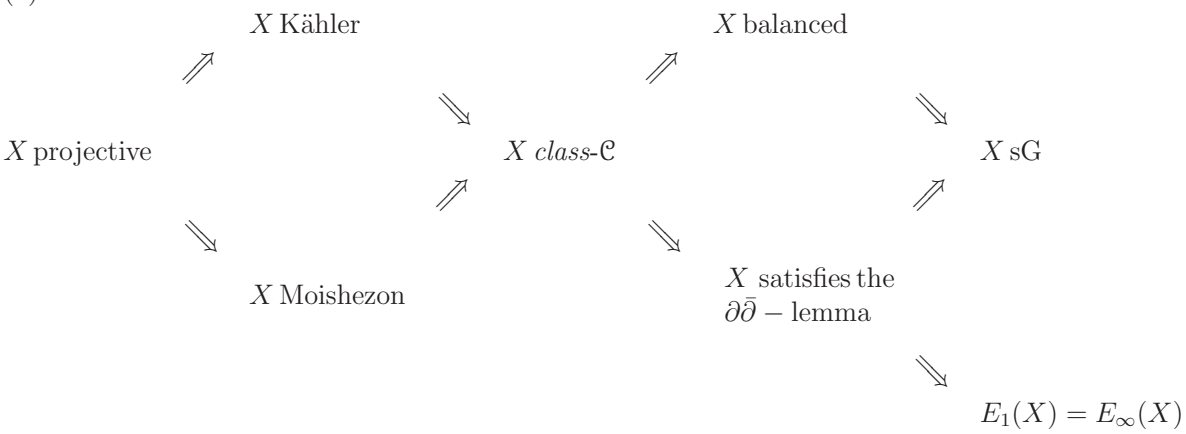

The above diagram simplifies dramatically, with many implications becoming equivalences, when $\operatorname{dim}_{\mathbb{C}} X=2$ :

$X$ projective $\Longleftrightarrow X$ Moishezon $\Longrightarrow X$ Kähler $\Longleftrightarrow X$ class $-\mathcal{C} \Longleftrightarrow X$ balanced $\Longleftrightarrow X$ satisfies the $\partial \bar{\partial}$-lemma $\Longleftrightarrow X \mathrm{sG}$;

and we always have $E_{1}(X)=E_{\infty}(X)$ for surfaces. 


\subsection{Examples}

One of the purposes of this work is to explain how the new notion of strongly Gauduchon manifold fits into the context of the well-known earlier other notions featuring in diagram $(\star)$ above. On one hand, we shall provide examples of compact complex manifolds that are not sG ( $c f$. Section 2) in order to stress that the $\mathrm{sG}$ condition is not automatically satisfied:

Theorem 1.9. The Calabi-Eckmann manifolds [12], the Hopf manifolds [26] and Tsuji's manifolds [46] are not strongly Gauduchon.

On the other hand, we shall provide examples of compact complex manifolds that drive home the peculiarity of the $\mathrm{SG}$ condition distinguishing it from the two stronger notions that immediately precede it in diagram $(\star)$ ( $c f$. also Theorem 3.15 in Section 3).

Theorem 1.10. There exist compact complex manifolds that are strongly Gauduchon but are not balanced and on which the $\partial \bar{\partial}$-lemma does not hold.

We also point out that the last two notions in diagram $(\star)$ are unrelated:

\section{Theorem 1.11.}

(a) There exist strongly Gauduchon compact complex manifolds whose Frölicher spectral sequence does not degenerate at $E_{1}$ (e.g. the Iwasawa manifold, which is even balanced).

(b) There exist compact complex manifolds whose Frölicher spectral sequence degenerates at $E_{1}$ but which are not strongly Gauduchon (e.g. any compact nonKähler complex surface).

This observation seems to indicate the existence of two disjoint realms in diagram $(\star)$ : the metric notions (i.e. balanced and $s G$ ) branching off upwards from the class- $\mathcal{C}$ notion; the topological notions (i.e. $\partial \bar{\partial}$-lemma and $E_{1}=E_{\infty}$ ) branching off downwards. The overall idea underlying the proof of our main result in [41] was based on switching from the latter to the former realm after observing that the topological condition of non-jumping at $t=0$ of the Hodge number $h^{0,1}(t)$ is sufficient for our purposes but next to impossible to guarantee on an a priori basis, we replaced it with the metric sG condition on the central fibre that we managed to guarantee.

\subsection{Holomorphic deformation behaviour}

Another purpose of this work is to review known results and open questions about the behaviour of these properties under holomorphic deformations, with a special emphasis on the new notion of strongly Gauduchon (or sG) manifolds. We adopt the Kodaira-Spencer terminology (cf.e.g. [28]):

A complex analytic (or holomorphic) family of compact complex manifods is a proper holomorphic submersion $\pi: \mathcal{X} \rightarrow \Delta$ from an arbitrary complex analytic manifold $\mathcal{X}$ to some open ball $\Delta \subset \mathbb{C}^{m}$ centered at the origin $0 \in \mathbb{C}^{m}$. 
Thus the fibres $X_{t}:=\pi^{-1}(t)$ for $t \in \Delta$, are all (smooth) compact complex manifolds of the same dimension varying holomorphically with the parameter $t \in$ $\Delta$. It is well-known that any such family is differentiably trivial, i.e. there exists a $C^{\infty}$ manifold $X$ independent of $t \in \Delta$ such that the fibre $X_{t}$ is $C^{\infty}$-diffeomorphic to $X$ for all $t \in \Delta$. Only the complex structure $J_{t}$ of $X_{t}$ varies with $t \in \Delta$. Thus the holomorphic family $\left(X_{t}\right)_{t \in \Delta}$ can be identified with a fixed $C^{\infty}$ manifold $X$ endowed with a holomorphic family of complex structures $\left(J_{t}\right)_{t \in \Delta}(c f$. [28]).

It will be sufficient to restrict our attention to the case where the base $\Delta$ is an open disc centered at the origin in $\mathbb{C}$, i.e. $m=1$. We shall be concerned with stability properties of the notions featuring in diagram $(\star)$ when they appear in holomorphic families as above. Two points of view will be adopted:

Definition 1.12. (i) A given property $(P)$ of a compact complex manifold is said to be open under holomorphic deformations if for every holomorphic family of compact complex manifolds $\left(X_{t}\right)_{t \in \Delta}$ and for every $t_{0} \in \Delta$, the following implication holds:

$X_{t_{0}}$ has property $(P) \Longrightarrow X_{t}$ has property $(P)$ for all $t \in \Delta$ sufficiently close to $t_{0}$.

(ii)A given property $(P)$ of a compact complex manifold is said to be closed under holomorphic deformations if for every holomorphic family of compact complex manifolds $\left(X_{t}\right)_{t \in \Delta}$ and for every $t_{0} \in \Delta$, the following implication holds:

$X_{t}$ has property $(P)$ for all $t \in \Delta \backslash\left\{t_{0}\right\} \Longrightarrow X_{t_{0}}$ has property $(P)$.

It is obvious that if a property $(P)$ is both open and closed, then all the fibres of a family satisfy $(P)$ whenever one of them satisfies $(P)$.

The interest in deformation stability questions was sparked by the following celebrated result of Kodaira and Spencer:

Theorem 1.13 (Kodaira-Spencer [29]). The Kähler property of compact complex manifolds is open under holomorphic deformations.

However, when Hironaka constructed an example in 1962, the following fact came as a bit of a surprise.

Theorem 1.14 (Hironaka [23]). The Kähler property of compact complex manifolds of complex dimension $\geq 3$ is not closed under holomorphic deformations.

Our main result of [41] states that the degeneration of the projective property of compact complex manifolds in the deformation limit is relatively mild, i.e. projective manifolds degenerate to Moishezon manifolds. In view of Hironoka's example that proved Theorem 1.14, the following result is optimal:

Theorem 1.15 (Theorem 1.1. in [41]). Let $\pi: \mathcal{X} \rightarrow \Delta$ be a complex analytic family of compact complex manifolds. If the fibre $X_{t}$ is projective for every $t \in \Delta^{\star}$, then $X_{0}$ is Moishezon. 
Hironaka's example does not cover the case of holomorphic families of compact complex surfaces, which turned out to behave very differently from manifolds of higher dimensions. Indeed, Kodaira's classification of surfaces, Miyaoka's result [36] asserting that an elliptic surface is Kähler if and only if its first Betti number is even, and Siu's result [45] asserting that every K3 surface is Kähler showed that the Kählerness of compact complex surfaces is a topological property:

Theorem 1.16 (Kodaira, Miyaoka, Siu). A compact complex surface $X$ is Kähler if and only if its first Betti number $b_{1}(X)$ is even.

Direct proofs of this theorem that do not invoke Kodaira's classification of compact complex surfaces were subsequently given by Buchdahl [9] and Lamari [31], independently. The reader will find further details on the history of the result in these references.

Since all the fibres $X_{t}$ in a holomorphic family of compact complex manifolds $\left(X_{t}\right)_{t \in \Delta}$ are differentiably diffeomorphic, they have the same Betti numbers. In particular, we have the following:

Corollary 1.17. The Kähler property of compact complex surfaces is both open and closed under holomorphic deformations. In particular, if in a holomorphic family of compact complex surfaces some fibre is Kähler, then all the fibres are Kähler.

Let us also recall that the deformation behaviour of the class-C property is the opposite of that of the Kähler property:

Theorem 1.18 (Campana [10], Lebrun-Poon [34]). The class $\mathcal{C}$ property of compact complex manifolds is not open under holomorphic deformations.

The examples proving this statement use families of twistor spaces and will be alluded to in section 4. Since it is known by a result of Campana [11] that the Moishezon and class- $\mathcal{C}$ properties are equivalent for twistor spaces, the Moishezon property is also seen not to be open under deformations. This latter fact is hardly surprising since a property associated with integral classes is not naturally expected to be deformation open. However, the following long-standing conjecture is still unsettled:

Conjecture 1.19. The class $\mathcal{C}$ property of compact complex manifolds is closed under holomorphic deformations.

Our main result in [42] amounts to a confirmation of this conjecture in the integral-class case, since Moishezon manifolds can be seen as integral-class versions of class- $\mathcal{C}$ manifolds (thanks to Theorems 1.1 and 1.3):

Theorem 1.20 ([42, Theorem 1.1.]). The Moishezon property ofcompact complex manifolds is closed under holomorphic deformations.

We have shown in [42] (see also Theorem 3.1 below) that the $s G$ property is deformation open. We hope that the following also holds: 
Conjecture 1.21. ${ }^{5}$ The sG property of compact complex manifolds is closed under holomorphic deformations.

The proof is still elusive, but if this turns out to be the case, the sG property would be the only known property of compact complex manifolds to be stable under all known operations (i.e. both open and closed under deformations, as well as stable under modifications as shown in $c f$. [43, Theorem 1.5 above]). In particular, it would suffice for just one fibre $X_{t_{0}}$ to be $\mathrm{sG}$ in order to guarantee that all the fibres $X_{t}$ are $\mathrm{sG}$. We have proved the conclusion of Conjecture 1.21 under a stronger assumption on the generic fibres and this has played a major part in our proofs of the main results of [41] and [42]:

Proposition 1.22 ( [41, Proposition 4.1.]). Let $\pi: \mathcal{X} \rightarrow \Delta$ be a complex analytic family of compact complex manifolds. If the $\partial \bar{\partial}$-lemma holds on $X_{t}$ for every $t \in$ $\Delta^{\star}$, then $X_{0}$ is a strongly Gauduchon manifold.

When it comes to balanced manifolds, Alessandrini and Bassanelli showed in [2] (see Theorem 3.3 below) that the balanced property of compact complex manifolds is not deformation open. This difference in deformation behaviour between $\mathrm{sG}$ and balanced manifolds will be exploited in Section 3. However, we hope that the balanced analogue of Conjecture 1.21 holds:

Conjecture 1.23. The balanced property of compact complex manifolds is closed under holomorphic deformations.

If this turns out to be the case, Conjecture 1.23 might be used to tackle the standard Conjecture 1.19. Indeed, by the Alessandrini-Bassanelli Theorem 1.4, proving that the limit fibre $X_{0}$ is balanced when $X_{t}$ has been supposed to be class $\mathcal{C}$ (hence also balanced) for all $t \neq 0$, is necessary to proving the stronger class $\mathcal{C}$ property of $X_{0}$.

As for the property of Frölicher degeneration at $E_{1}$, we have the following:

Theorem 1.24. (a) [29, Kodaira-Spencer] For compact complex manifolds, the property of the Frölicher spectral sequence degenerating at $E_{1}$ is open under holomorphic deformations.

(b) (Eastwood-Singer [16, Theorem 5.4.]) For compact complex manifolds, the property of the Frölicher spectral sequence degenerating at $E_{1}$ is not closed under holomorphic deformations.

Part (a) is by now a classical statement that follows immediately from the inequality (1.1) satisfied by every fibre $X_{t}$ (in which equality is equivalent to $E_{1}\left(X_{t}\right)=$ $E_{\infty}\left(X_{t}\right)$ ), from the Betti numbers $b_{k}\left(X_{t}\right)$ of the fibres being independent of $t$ (thanks to the $C^{\infty}$ triviality of the family) and from the upper-semicontinuity of every Hodge number $h^{p, q}\left(X_{t}\right)$ with respect to $t \in \Delta$ (which is another classical

5 This conjecture was a posteriori suggested to the author by Jean-Pierre Demailly as a reinforcement of our earlier Proposition 1.22. 
result of Kodaira and Spencer). An overview of the Eastwood-Singer proof of part (b) will be given in Section 4.

Turning now to the $\partial \bar{\partial}$-lemma, we have the following:

Theorem 1.25 ([49, C.-C. Wu]). ${ }^{6}$ For compact complex manifolds, the $\partial \bar{\partial}$-lemma property is open under holomorphic deformations. section.

We give a brief account of Wu's strategy of proof in Subsection4.3 of the last

We are at a loss to know anything about the deformation limits of compact complex manifolds satisfying the $\partial \bar{\partial}$-lemma. It might well be the case that this property is not deformation-closed although the current evidence is tenuous. We will outline a possible approach to this question via twistor spaces in Subsection 4.4 of the last Section 4.

ACKnOwledgements. The author is extremely grateful to Professor Akira Fujiki for kindly inviting him to Osaka University, for patiently explaining to him exciting titbits about various notions in mathematics and for indicating a host of bibliographical references that have broadened his understanding of a wider picture. Thanks are also due to Professor Hajime Tsuji over a similar invitation to Tokyo and for kindly pointing out the reference [46].

\section{Examples of non-sG compact complex manifolds}

In this section we prove Theorem 1.9 by pointing out three well-known classes of compact complex manifolds as not being sG: the Calabi-Eckmann manifolds, the Hopf manifolds and Tsuji's manifolds constructed in [46]. The underlying space of all these manifolds is a product $X:=S^{2 p+1} \times S^{2 q+1}$ of two real odd-dimensional spheres, so they all share the property $H_{D R}^{2}(X, \mathbb{R})=0$ for the second De Rham cohomology group. This implies that any $d$-closed positive current $T$ of type $(1,1)$ on $X$, should it exist, must be $d$-exact since the associated De Rham cohomology 2class $\{T\} \in H_{D R}^{2}(X, \mathbb{R})$ must vanish. The existence of a non-trivial $(1,1)$-current $T$ on $X$ that is both positive and $d$-exact amounts to $X$ being non-sG as the following intrinsic characterisation of $\mathrm{sG}$ manifolds obtained in [41] shows:

Proposition 2.1 ([41, Proposition 3.3.]). Let $X$ be a compact complex manifold. Then $X$ carries a strongly Gauduchon metric if and only if there exists no non-zero current $T$ of type $(1,1)$ such that $T \geq 0$ and $T$ is $d$-exact on $X$.

We shall briefly review the three classes of compact complex manifolds mentioned above and notice that every such manifold $X$ possesses complex hypersurfaces $Y \subset X$. Thus, since $H_{D R}^{2}(X, \mathbb{R})=0$, the current of integration on any of

6 The author is grateful to Professor Jixiang Fu for pointing out to him this work of Wu of which he was unfortunately unaware when writing the first version of the present paper. 
these complex hypersurfaces $Y$ is a current as in Proposition 2.1, ruling out the possibility that any manifold $X$ in one of these classes be sG.

(a) Calabi-Eckmann manifolds. For all $p, q \in \mathbb{N}$, Calabi and Eckmann [12] constructed a complex structure on the Cartesian product $S^{2 p+1} \times S^{2 q+1}$ of odddimensional spheres. The case $p=q=0$ being equivalent to a closed Riemann surface of genus 1 and periods $1, \tau$, they assume $p>0$. In the case $q=0$, the Calabi-Eckmann complex structure on $S^{2 p+1} \times S^{1}$, although constructed by a different method, coincides with the complex structure constructed earlier by Hopf in [26] starting from the universal covering space of $S^{2 p+1} \times S^{1}$ equipped with the complex structure of $\mathbb{C}^{p+1} \backslash\{0\}$. The simply connected manifolds $S^{2 p+1} \times S^{2 q+1}$ $(p, q>0)$ are given in [12] complex structures making them into compact, simply connected, non-Kähler complex manifolds $M^{p, q}$ of complex dimension $p+q+1$ enjoying, among other things, the following properties (for all $p, q$, including $q=$ $0)$ :

(i) There exists a complex analytic fibring $\sigma: M^{p, q} \rightarrow \mathbb{P}^{p} \times \mathbb{P}^{q}$ over the product of complex projective spaces $\mathbb{P}^{p}$ and $\mathbb{P}^{q}$ whose fibres are tori of real dimension 2 (or algebraic curves of genus 1) ( $c f$. [12, Theorem II]);

(ii) Every compact complex subvariety of $M^{p, q}$ is the set of all points that are mapped by $\sigma$ onto an algebraic subvariety of $\mathbb{P}^{p} \times \mathbb{P}^{q}$; it is therefore also fibred by tori ( $c f .[12$, Theorem IV]).

It is clear that the inverse image under $\sigma$ of any complex hypersurface of $\mathbb{P}^{p} \times \mathbb{P}^{q}$ defines a complex hypersurface of the Calabi-Eckmann manifold $M^{p, q}$. Thus no Calabi-Eckmann manifold $M^{p, q}(p>0)$ can be an $\mathrm{sG}$ manifold. ${ }^{7}$

(b) Hopf manifolds. As mentioned above (and proved in [12, Section 3]), the Hopf manifolds $S^{2 p+1} \times S^{1}(p>0)$ endowed with the complex structure constructed in [Hop48] can be seen in retrospect as special cases for $q=0$ of Calabi-Eckmann manifolds. Thus they contain complex hypersurfaces and are not sG manifolds by the above arguments.

(c) Tsuji's manifolds. Generalising the Calabi-Eckmann complex structures, Tsuji constructed in [46] complex structures on $S^{3} \times S^{3}$ in the following way. Starting from an arbitrary $\left(\alpha_{1}, \alpha_{2}, \alpha_{3}\right) \in \mathbb{C}^{3}$ satisfying

$$
0<\left|\alpha_{1}\right| \leq\left|\alpha_{2}\right|<1 \text { and } 0<\left|\alpha_{3}\right|<1 \text {, }
$$

the author of [46] considers the primary Hopf manifold

$$
H(\alpha):=\mathbb{C}^{3} \backslash\{0\} /\langle h\rangle
$$

7 This same argument was invoked in [35, page 263], to show that Calabi-Eckmann manifolds are not balanced. 
of complex dimension 3, where the automorphism $h: \mathbb{C}^{3} \rightarrow \mathbb{C}^{3}$ is defined by $h\left(z_{1}, z_{2}, z_{3}\right):=\left(\alpha_{1} z_{1}, \alpha_{2} z_{2}, \alpha_{3} z_{3}\right)$ for all $\left(z_{1}, z_{2}, z_{3}\right) \in \mathbb{C}^{3}$ and $\langle h\rangle \subset \operatorname{Aut}\left(\mathbb{C}^{3}\right)$ denotes the automorphism group generated by $h$. He then goes on to consider

$$
C:=\left\{\left[z_{1}, z_{2}, z_{3}\right] \in H(\alpha) ; z_{1}=z_{2}=0\right\} \subset H(\alpha),
$$

an elliptic curve contained in $H(\alpha)$ and

$$
S_{0}:=\left\{\left[z_{1}, z_{2}, z_{3}\right] \in H(\alpha) ; z_{3}=0\right\} \subset H(\alpha),
$$

a primary Hopf surface which is a complex hypersurface of $H(\alpha)$. For every

$$
A=\left(\begin{array}{ll}
a & b \\
c & d
\end{array}\right) \in S L(2, \mathbb{Z}) \text { and } m=\left(m_{1}, m_{2}\right) \in \mathbb{Z}^{2}, m_{1}, m_{2} \gg 1,
$$

he shows the existence of $\beta=\left(\beta_{1}, \beta_{2}, \beta_{3}\right) \in \mathbb{C}^{3}$ defining biholomorphisms

$$
L^{\star}(\beta) \stackrel{\Phi^{ \pm}}{\simeq} L^{\star}(\alpha),
$$

where $L^{\star}(\alpha)$ and $L^{\star}(\beta)$ are obtained from $L(\alpha)$ and $L(\beta)$ by removing the respective zero section, while $L(\alpha)$ and $L(\beta)$ are holomorphic line bundles over the respective primary Hopf surfaces

$$
S_{\alpha_{1}, \alpha_{2}, 0}:=\mathbb{C}^{2} \backslash\{0\} /\left\langle g_{\alpha}\right\rangle \text { and } S_{\beta_{1}, \beta_{2}, 0}:=\mathbb{C}^{2} \backslash\{0\} /\left\langle g_{\beta}\right\rangle
$$

associated with automorphisms of $\mathbb{C}^{2}$

$$
g_{\alpha}\left(z_{1}, z_{2}\right):=\left(\alpha_{1} z_{1}, \alpha_{2} z_{2}\right) \text { and } g_{\beta}\left(z_{1}, z_{2}\right):=\left(\beta_{1} z_{1}, \beta_{2} z_{2}\right)
$$

defined by

$$
\begin{aligned}
& L(\alpha):=\mathbb{C}^{2} \backslash\{0\} \times \mathbb{C} /\left\langle h_{\alpha}\right\rangle \\
& L(\beta):=\mathbb{C}^{2} \backslash\{0\} \times \mathbb{C} /\left\langle h_{\beta}\right\rangle,
\end{aligned}
$$

where the automorphisms $h_{\alpha}$ and $h_{\beta}$ of $\mathbb{C}^{3}$ are defined by

$$
\begin{aligned}
& h_{\alpha}\left(z_{1}, z_{2}, z_{3}\right):=\left(\alpha_{1} z_{1}, \alpha_{2} z_{2}, \alpha_{3} z_{3}\right) \\
& h_{\beta}\left(z_{1}, z_{2}, z_{3}\right):=\left(\beta_{1} z_{1}, \beta_{2} z_{2}, \beta_{3} z_{3}\right) .
\end{aligned}
$$

Considering a compactification of $L(\beta)$ as a $\mathbb{P}^{1}$-bundle $\mathbb{P}(\beta) \rightarrow S_{\beta_{1}, \beta_{2}, 0}$, the infinity section of $\mathbb{P}(\beta)$ is denoted $S_{\infty}$, while $U\left(S_{\infty}\right)$ denotes a tubular neighbourhood of $S_{\infty}$ in $\mathbb{P}(\beta)$. The author defines compact complex manifolds

$$
M^{ \pm}(\alpha, A, m)
$$

by identifying

$$
L^{\star}(\beta) \subset \mathbb{P}(\beta) \backslash \text { (zero section) }
$$

with

$$
L^{\star}(\alpha) \simeq H(\alpha) \backslash\left(S_{0} \cup C\right) \subset H(\alpha)
$$


using $\Phi^{ \pm}$. These compact complex manifolds are seen to arise as

$$
M^{ \pm}(\alpha, A, m)=(H(\alpha) \backslash C) \cup U\left(S_{\infty}\right),
$$

or equivalently, $M^{ \pm}(\alpha, A, m)$ are obtained from $H(\alpha)$ by a surgery which replaces $C$ with $U\left(S_{\infty}\right)$.

Theorem 2.2 ( [46, Theorem 1.13]). $M^{ \pm}(\alpha, A, m)$ is diffeomorphic to $S^{3} \times S^{3}$ if and only if $A$ is of the form $A=\left(\begin{array}{cc}a & b \\ \pm 1 & d\end{array}\right)$.

Consequently, if $A$ has the above shape, $M^{ \pm}(\alpha, A, m)$ is diffeomorphic to an $S^{3}$-bundle over a lens space, hence $M^{ \pm}(\alpha, A, m)$ has a complex structure.

With this outline of Tsuji's construction understood, we see that the complex hypersurface $S_{0} \subset H(\alpha)$ satisfies $S_{0} \cap C=\emptyset$. Thus, in view of the description (2.1) of $M^{ \pm}(\alpha, A, m)$, we get a complex hypersurface

$$
S_{0} \subset M^{ \pm}(\alpha, A, m)
$$

whose existence, along with the property $H_{D R}^{2}\left(M^{ \pm}(\alpha, A, m), \mathbb{R}\right)=0$, shows that Tsuji's compact complex manifolds $M^{ \pm}(\alpha, A, m)$ are not sG for any $\alpha \in \mathbb{C}^{3}, A \in$ $S L(2, \mathbb{Z}), m=\left(m_{1}, m_{2}\right) \in \mathbb{Z}^{2}$ as above.

\section{Examples of sG manifolds}

As pointed out in the Introduction, all balanced manifolds and all compact complex manifolds on which the $\partial \bar{\partial}$-lemma holds provide examples of $\mathrm{sG}$ manifolds $(c f$. implication diagram $(\star))$. In this section we shall exhibit compact complex manifolds that are $\mathrm{sG}$ but neither are they balanced nor do they satisfy the $\partial \bar{\partial}$-lemma. These examples are thus meant to emphasise the difference between $\mathrm{sG}$ manifolds and stronger earlier types of possibly non-Kähler compact complex manifolds.

\subsection{Deformation stability}

The examples we shall exhibit will be provided by holomorphic families of compact complex manifolds. The starting point is the following stability property of $s G$ manifolds under small deformations:

Theorem 3.1 ([42, Conclusion 2.4.]). The sG property of compact complex manifolds is open under holomorphic deformations.

We now recall the proof of this small deformation stability result. The main argument is provided by the following characterisation of $\mathrm{sG}$ manifolds:

Lemma 3.2 ([41, Lemma 3.2]). Let $X$ be a compact complex manifold of complex dimension $n$. Then $X$ carries an $s G$ metric if and only if there exists $a C^{\infty}(2 n-2)$ form $\Omega$ on $X$ satisfying the following three conditions:

(a) $\Omega=\bar{\Omega}$ (i.e. $\Omega$ is real);

(b) $d \Omega=0$; 
(c) $\Omega^{n-1, n-1}>0$ on $X$ (i.e. the component of type $(n-1, n-1)$ of $\Omega$ with respect to the complex structure of $X$ is positive-definite).

Note that conditions (a) and (b) are independent of the complex structure of $X$, while a change of complex structure changes the $(n-1, n-1)$-component of a given $(2 n-2)$-form $\Omega$. Thus condition (c) is the only one to be dependent on the complex structure of $X$.

Proof of Lemma 3.2 (cf. [41]). The vanishing of the ( $2 n-1)$-form $d \Omega(c f$. (b)) amounts to the simultaneous vanishing of its components $\partial \Omega^{n-1, n-1}+\bar{\partial} \Omega^{n, n-2}$ (of type $(n, n-1)$ ) and $\partial \Omega^{n-2, n}+\bar{\partial} \Omega^{n-1, n-1}$ (of type $(n-1, n)$ ). These two components are conjugate to each other if $\Omega$ satisfies (a). Thus, if (a) holds, (b) is equivalent to $\partial \Omega^{n-1, n-1}+\bar{\partial} \Omega^{n, n-2}=0$.

Suppose there exists an sG metric $\omega$ on $X$. This means that $\omega$ is a $C^{\infty}$ positivedefinite $(1,1)$-form on $X$ such that the $(n, n-1)$-form $\partial \omega^{n-1}$ is $\bar{\partial}$-exact. Then the $(n-1, n-1)$-form $\Omega^{n-1, n-1}:=\omega^{n-1}$ is positive-definite on $X$ and there exists a $C^{\infty}(n, n-2)$-form $\Omega^{n, n-2}$ on $X$ satisfying $\partial \Omega^{n-1, n-1}=-\bar{\partial} \Omega^{n, n-2}$. Considering the $(n-2, n)$-form $\Omega^{n-2, n}:=\overline{\Omega^{n, n-2}}$, we see that the $C^{\infty}(2 n-2)$-form

$$
\Omega:=\Omega^{n, n-2}+\Omega^{n-1, n-1}+\Omega^{n-2, n}
$$

satisfies conditions (a), (b), (c).

Conversely, suppose there exists a $C^{\infty}(2 n-2)$-form $\Omega$ on $X$ satisfying conditions (a), (b), (c). According to an observation in linear algebra due to Michelsohn [35], every $C^{\infty}$ positive-definite $(n-1, n-1)$-form admits a unique $(n-1)^{s t}$ root. Applying this to $\Omega^{n-1, n-1}>0$, we get a unique $C^{\infty}$ positive-definite $(1,1)$ form $\omega>0$ on $X$ such that

$$
\omega^{n-1}=\Omega^{n-1, n-1} .
$$

By condition $(b)$ satisfied by $\Omega$, we see that $\partial \omega^{n-1}$ is $\bar{\partial}$-exact, which means that the Hermitian metric $\omega$ of $X$ is strongly Gauduchon.

Proof of Theorem 3.1. If we are given a holomorphic family of compact complex manifolds $\left(X_{t}\right)_{t \in \Delta}$ with $\operatorname{dim}_{\mathbb{C}} X_{t}=n$, we denote by $X$ the $C^{\infty}$ manifold underlying the fibres $X_{t}$ and by $J_{t}$ the complex structure of $X_{t}$ for all $t \in \Delta$. The family $\left(X_{t}\right)_{t \in \Delta}$ is thus equivalent to the holomorphic family of complex structures $\left(J_{t}\right)_{t \in \Delta}$ on $X$. If we have a $C^{\infty}(2 n-2)$-form $\Omega$ on $X$, its components $\Omega_{t}^{n-1, n-1}$ of type $(n-1, n-1)$ with respect to the complex structures $J_{t}$ vary in a $C^{\infty}$ way with $t \in \Delta$. Consequently, if $\Omega_{0}^{n-1, n-1}>0$ then $\Omega_{t}^{n-1, n-1}>0$ for $t \in \Delta$ sufficiently close to $0 \in \Delta$. Thus condition (c) of Lemma 3.2 is preserved under small deformations by mere continuity. Since conditions (a) and (b) of Lemma 3.2 are independent of the complex structure of $X$, it follows that any $C^{\infty}(2 n-2)$-form $\Omega$ on $X$ satisfying conditions (a), (b) and (c) of Lemma 3.2 with respect to $J_{0}$ also satisfies these conditions with respect to $J_{t}$ for all $t$ sufficiently near 0 . The proof of Theorem 3.1 is complete. 
One fundamental difference between balanced and sG manifolds that we shall exploit is that, unlike sG manifolds, balanced manifolds are not stable under small deformations. This result was first observed by Alessandrini and Bassanelli [2] and refutes Michelsohn's claim of the contrary made in the introduction to [35]:

Theorem 3.3 (Alessandrini-Bassanelli [2]). The balanced property of compact complex manifolds is not open under holomorphic deformations.

\subsection{The Iwasawa manifold}

Alessandrini and Bassanelli use the explicit description of the Kuranishi family of the Iwasawa manifold (known to be balanced) calculated by Nakamura in [37] and observe that one particular direction among the six dimensions of the base space yields the example that proves Theorem 3.3. We now review a few basic facts about compact complex parallelisable manifolds to the class of which the Iwasawa manifold belongs, before surveying the arguments and results of [2,37] and [3] that are necessary to the understanding of the observation of Alessandrini and Bassanelli (cf. Proposition 3.13) which proves Theorem 3.3.

Let $X$ be a compact complex manifold, $\operatorname{dim}_{\mathbb{C}} X=n$. Recall the following:

Fact. If $X$ is Kähler, then for every $p=0,1, \ldots, n$ and for every form $u \in$ $C^{\infty}\left(X, \Lambda^{p, 0} T^{\star} X\right)$ such that $\bar{\partial} u=0$, we have $d u=0$. In other words, every holomorphic $p$-form is $d$-closed on a compact Kähler manifold.

To see this, fix any Kähler metric $\omega$ on $X$ and recall that the associated $d$ Laplacian $\Delta_{\omega}:=d d^{\star}+d^{\star} d$, $\partial$-Laplacian $\Delta_{\omega}^{\prime}:=\partial \partial^{\star}+\partial^{\star} \partial$ and $\bar{\partial}$-Laplacian $\Delta_{\omega}^{\prime \prime}:=\bar{\partial} \bar{\partial}^{\star}+\bar{\partial}^{\star} \bar{\partial}$ (where the formal adjoints $d^{\star}, \partial^{\star}, \bar{\partial}^{\star}$ are calculated with respect to $\omega)$ are related by

$$
\Delta_{\omega}^{\prime}=\Delta_{\omega}^{\prime \prime}=\frac{1}{2} \Delta_{\omega}
$$

This property is peculiar to Kähler metrics; it fails dramatically for arbitrary, nonKähler metrics. Now, given any smooth $(p, 0)$-form $u$ on $X$, we clearly have $\bar{\partial}^{\star} u=$ 0 for trivial bidegree reasons. If $u$ is holomorphic (i.e. $\bar{\partial} u=0)$, then $\Delta_{\omega}^{\prime \prime} u=0$ because $\operatorname{ker} \Delta_{\omega}^{\prime \prime}=\operatorname{ker} \bar{\partial} \cap \operatorname{ker} \bar{\partial}^{\star}$. (This last identity of kernels is valid for any Hermitian metric $\omega$ on a compact manifold.) It then follows from (3.1) that $\Delta_{\omega} u=$ 0 . Since $\operatorname{ker} \Delta_{\omega}=\operatorname{ker} d \cap \operatorname{ker} d^{\star}$, we see that $d u=0$.

Let us now recall that this standard fact enables one to see that the Iwasawa manifold is not Kähler. The Iwasawa manifold is, by definition, the compact complex manifold of complex dimension 3 defined as the quotient

$$
X:=G / \Gamma
$$

of the simply connected, connected complex Lie group (the Heisenberg group)

$$
G:=\left\{\left(\begin{array}{ccc}
1 & z_{1} & z_{3} \\
0 & 1 & z_{2} \\
0 & 0 & 1
\end{array}\right) ; z_{1}, z_{2}, z_{3} \in \mathbb{C}\right\} \subset \mathrm{Gl}_{3}(\mathbb{C})
$$


by the discrete subgroup $\Gamma \subset G$ of matrices with entries $z_{1}, z_{2}, z_{3} \in \mathbb{Z}[i]$. The complex manifold structure on $G$ is defined by the complex structure of $\mathbb{C}^{3}$ via the obvious diffeomorphism $G \simeq \mathbb{C}^{3}$, while the group structure on $G$ is defined by the multiplication of matrices

$$
\left(\begin{array}{ccc}
1 & z_{1} & z_{3} \\
0 & 1 & z_{2} \\
0 & 0 & 1
\end{array}\right)\left(\begin{array}{ccc}
1 & w_{1} & w_{3} \\
0 & 1 & w_{2} \\
0 & 0 & 1
\end{array}\right)=\left(\begin{array}{ccc}
1 & z_{1}+w_{1} & z_{3}+w_{3} \\
0 & 1 & z_{2}+w_{2} \\
0 & 0 & 1
\end{array}\right)
$$

Since the holomorphic 1-form on $G$

$$
G \ni M \mapsto M^{-1} d M
$$

is invariant under the action of $\Gamma$, it descends to a holomorphic 1-form on $X$. An elementary calculation shows that

$$
\text { if } \quad M=\left(\begin{array}{ccc}
1 & z_{1} & z_{3} \\
0 & 1 & z_{2} \\
0 & 0 & 1
\end{array}\right) \text { then } M^{-1} d M=\left(\begin{array}{ccc}
0 & d z_{1} & d z_{3}-z_{1} d z_{2} \\
0 & 0 & d z_{2} \\
0 & 0 & 0
\end{array}\right)
$$

Thus we get holomorphic 1 -forms on the Iwasawa manifold $X$ induced by the following forms on $\mathbb{C}^{3}$ :

$$
\varphi_{1}:=d z_{1}, \quad \varphi_{2}:=d z_{2}, \quad \varphi_{3}:=d z_{3}-z_{1} d z_{2} .
$$

Denoting the induced forms by the same symbols $\varphi_{1}, \varphi_{2}, \varphi_{3}$, it is obvious that

$$
d \varphi_{1}=d \varphi_{2}=0 \quad \text { but } d \varphi_{3}=-\varphi_{1} \wedge \varphi_{2} \neq 0 \quad \text { on } X \text {. }
$$

Since the holomorphic 1 -form $\varphi_{3}$ on $X$ is not $d$-closed, $X$ is not Kähler.

Finally recall that the forms defined in (3.2), which are linearly independent at every point of $X$, can be used to completely calculate the De Rham, Dolbeault and Bott-Chern cohomologies of the Iwasawa manifold (see e.g. [44, page 4-6] for details). For example, $\varphi_{1}, \varphi_{2}, \overline{\varphi_{1}}, \overline{\varphi_{2}}$ are all $d$-closed, but not $d$-exact, 1 -forms on $X$ and any two of them are not $d$-cohomologous. For instance, if we had $\varphi_{1}=$ $d f=\partial f+\bar{\partial} f$ for a smooth function $f$ on $X$, then $\bar{\partial} f=0$ since $\varphi_{1}$ is of type $(1,0)$. Thus $f$ would be holomorphic, hence constant, on the compact $X$, which is impossible since $\varphi_{1}$ is not zero. It is readily seen that $H_{D R}^{1}(X, \mathbb{C})$ is the $\mathbb{C}$-vector space generated as follows:

$$
H_{D R}^{1}(X, \mathbb{C})=\left\langle\left\{\varphi_{1}\right\},\left\{\varphi_{2}\right\},\left\{\overline{\varphi_{1}}\right\},\left\{\overline{\varphi_{2}}\right\}\right\rangle, \text { hence } b_{1}(X)=4
$$

where \{ $\}$ denotes a De Rham cohomology class. Using (3.3), we can easily see that $\varphi_{1} \wedge \varphi_{3}$ and $\varphi_{2} \wedge \varphi_{3}$ (both of type $(2,0)$ ) are $d$-closed but not $d$-exact on $X$, hence they and their conjugates (the latter being of type $(0,2))$ induce non-zero elements in $H_{D R}^{2}(X, \mathbb{C})$, while $\varphi_{1} \wedge \varphi_{2}$ is $d$-exact on $X$ hence it induces the zero 
class. On the other hand, the $(1,1)$-forms $\varphi_{1} \wedge \overline{\varphi_{1}}, \varphi_{2} \wedge \overline{\varphi_{2}}, \varphi_{1} \wedge \overline{\varphi_{2}}$ and $\varphi_{2} \wedge \overline{\varphi_{1}}$ are all $d$-closed but not $d$-exact. We easily get that $H_{D R}^{2}(X, \mathbb{C})$ is the $\mathbb{C}$-vector space generated as follows:

$$
\begin{aligned}
H_{D R}^{2}(X, \mathbb{C})= & \left\langle\left\{\varphi_{1} \wedge \varphi_{3}\right\},\left\{\varphi_{2} \wedge \varphi_{3}\right\}\right\rangle \\
& \oplus\left\langle\left\{\varphi_{1} \wedge \overline{\varphi_{1}}\right\},\left\{\varphi_{2} \wedge \overline{\varphi_{2}}\right\},\left\{\varphi_{1} \wedge \overline{\varphi_{2}}\right\},\left\{\varphi_{2} \wedge \overline{\varphi_{1}}\right\}\right\rangle \\
& \left.\oplus\left\langle\left\{\overline{\varphi_{1}} \wedge \overline{\varphi_{3}}\right\},\left\{\overline{\varphi_{2}} \wedge \overline{\varphi_{3}}\right\}\right\}\right\rangle, \quad \text { hence } b_{2}(X)=8 .
\end{aligned}
$$

We have recalled (3.4) and (3.5) since they will be used further on.

We now review the argument showing that the Iwasawa manifold is balanced. Actually every compact complex parallelisable manifold will be seen to be balanced. The point of view presented here is that of [3].

Observation 3.4 (cf.e.g. [37, Lemma 1.2.] or [3, Remark 3.1.]). Let $X$ be any compact complex (possibly non-Kähler) manifold, $\operatorname{dim}_{\mathbb{C}} X=n$. Then for every form $u \in C^{\infty}\left(X, \Lambda^{n-1,0} T^{\star} X\right)$ such that $\bar{\partial} u=0$, we have $d u=0$. In other words, every holomorphic $(n-1)$-form is $d$-closed on any compact complex manifold of dimension $n$.

Proof. Let $u \in C^{\infty}\left(X, \Lambda^{n-1,0} T^{\star} X\right)$ such that $\bar{\partial} u=0$. Then $d u=\partial u$ is of type $(n, 0)$ and $d \bar{u}=\overline{d u}=\overline{\partial u}$ is of type $(0, n)$. We get

$$
i^{n^{2}} d u \wedge d \bar{u} \geq 0 \quad \text { as an }(n, n)-\text { form on } \quad X
$$

and

$$
\int_{X} i^{n^{2}} d u \wedge d \bar{u}=i^{n^{2}} \int_{X} d(u \wedge d \bar{u})=0 \text { by Stokes. }
$$

Thus (3.6) and (3.7) yield $i^{n^{2}} d u \wedge d \bar{u}=0$ everywhere on $X$, hence $d u=0$ everywhere on $X$. This proves the contention. To justify (3.6), write in local holomorphic coordinates $z_{1}, \ldots, z_{n}$ :

$d u=f d z_{1} \wedge \ldots d z_{n}, \quad$ hence $i^{n^{2}} d u \wedge d \bar{u}=|f|^{2} i d z_{1} \wedge d \bar{z}_{1} \ldots i d z_{n} \wedge d \bar{z}_{n} \geq 0$.

It is clear that $i^{n^{2}} d u \wedge d \bar{u}=0$ iff $f=0$ iff $d u=0$.

Corollary 3.5. Let $X$ be a compact complex manifold, $\operatorname{dim}_{\mathbb{C}} X=n$. Suppose we have a form $u \in C^{\infty}\left(X, \Lambda^{n-1,0} T^{\star} X\right)$ such that $\bar{\partial} u=0$.

Then the $(n-1, n-1)$-form $i^{(n-1)^{2}} u \wedge \bar{u}$ satisfies

$$
i^{(n-1)^{2}} u \wedge \bar{u} \geq 0 \quad \text { and } \quad d\left(i^{(n-1)^{2}} u \wedge \bar{u}\right)=0 \text { on } X .
$$

Proof. The first inequality is checked to hold for any $(n-1,0)$-form $u$ by a trivial calculation. If $\bar{\partial} u=0$, then $d u=0$ by Observation 3.4. Then we also have $d \bar{u}=0$ and the second part follows. 
Now recall the following standard notion introduced by Wang [Wan54]. A compact complex manifold $X$ is said to be complex parallelisable if its holomorphic tangent bundle $T^{1,0} X$ is trivial. This condition is, of course, equivalent to the sheaf of germs of holomorphic 1-forms $\Omega_{X}^{1}$ being trivial. If $n=\operatorname{dim}_{\mathbb{C}} X$, the complex parallelisable condition is equivalent to the existence of $n$ holomorphic vector fields $\theta_{1}, \ldots, \theta_{n} \in H^{0}\left(X, T^{1,0} X\right)$ that are linearly independent at every point of $X$. It is again equivalent to the existence of $n$ holomorphic 1 -forms $\varphi_{1}, \ldots, \varphi_{n} \in H^{0}\left(X, \Omega_{X}^{1}\right)$ that are linearly independent at every point of $X$.

By a result of Wang [48], every compact complex parallelisable manifold is the compact quotient $X=G / \Gamma$ of a simply connected, connected complex Lie group $G$ by a discrete subgroup $\Gamma \subset G$. Conversely, it is obvious that any such quotient is complex parallelisable. In particular, for any compact complex parallelisable manifold $X, H^{0}\left(X, T^{1,0} X\right) \simeq \mathfrak{g}$ where $\mathfrak{g}$ is the Lie algebra of $G$. A compact complex parallelisable manifold $X$ is said to be nilpotent (respectively solvable) ${ }^{8}$ if the corresponding complex Lie group $G$ is nilpotent (respectively solvable). The Heisenberg group defining the Iwasawa manifold being nilpotent, the Iwasawa manifold is a nilpotent compact complex parallelisable manifold.

Corollary 3.6 (cf. e.g. [3, Remark 3.1.]). Every compact complex parallelisable manifold is balanced.

In particular, the Iwasawa manifold is balanced.

Proof. Let $X$ be an arbitrary compact complex parallelisable manifold, $\operatorname{dim}_{\mathbb{C}} X=$ $n$. Let $\varphi_{1}, \ldots, \varphi_{n} \in H^{0}\left(X, \Omega_{X}^{1}\right)$ be $n$ holomorphic 1-forms that are linearly independent at every point of $X$. Consider the $(n-1, n-1)$-form on $X$ :

$\Omega:=i^{(n-1)^{2}} \sum_{i=1}^{n} \varphi_{1} \wedge \cdots \wedge \widehat{\varphi_{i}} \wedge \cdots \wedge \varphi_{n} \wedge \bar{\varphi}_{1} \wedge \cdots \wedge \widehat{\bar{\varphi}_{i}} \wedge \cdots \wedge \bar{\varphi}_{n}=\sum_{i=1}^{n} i^{(n-1)^{2}} u_{i} \wedge \bar{u}_{i}$

where $u_{i}:=\varphi_{1} \wedge \cdots \wedge \widehat{\varphi_{i}} \wedge \cdots \wedge \varphi_{n} \in C^{\infty}\left(X, \Lambda^{n-1,0} T^{\star} X\right)$ and indicates a missing factor. Since $\bar{\partial} \varphi_{k}=0$ for all $k=1, \ldots, n$, we see that $\bar{\partial} u_{i}=0$ for all $i=1, \ldots, n$. Then Observation 3.4 gives $d u_{i}=0$ for all $i=1, \ldots, n$, while Corollary 3.5 gives

$$
\Omega \geq 0 \text { and } d \Omega=0 \text { on } X \text {. }
$$

Furthermore, since $\varphi_{1}, \ldots, \varphi_{n}$ are linearly independent at every point of $X$, we must even have $\Omega>0$. Thus $\Omega$ is a $C^{\infty}(n-1, n-1)$-form on $X$ satisfying

$$
\Omega>0 \text { and } d \Omega=0 \text { on } X \text {. }
$$

Applying Michelsohn's procedure [35] for extracting the $(n-1)^{s t}$ root of a smooth positive-definite $(n-1, n-1)$-form, there exists a unique $C^{\infty}$ positive-definite $(1,1)$-form $\omega>0$ on $X$ such that $\omega^{n-1}=\Omega$. Since $d\left(\omega^{n-1}\right)=d \Omega=0$, we see that $\omega$ is a balanced metric on $X$. The proof is complete.

${ }^{8}$ Or to be a compact complex parallelisable nilmanifold (respectively solvmanifold). 
Note, however, that very few compact complex parallelisable manifolds are Kähler, thanks to a result of Wang:

Remark 3.7 ([48, Corollary 2, page 776]). Let $X=G / \Gamma$ be a compact complex parallelisable manifold. Then

$X$ is Kähler $\Longleftrightarrow \quad G$ is Abelian $\Longleftrightarrow X$ is a complex torus.

\subsection{The Kuranishi family of the Iwasawa manifold (after Nakamura [37])}

(a) Let $X$ be a compact complex manifold, with $\operatorname{dim}_{\mathbb{C}} X=n$. Since there are no non-zero $\bar{\partial}$-exact $(1,0)$-forms on $X$ (for obvious bidegree reasons), we have

$$
H^{1,0}(X, \mathbb{C})=\left\{u \in C^{\infty}\left(X, \Lambda^{1,0} T^{\star} X\right) ; \bar{\partial} u=0\right\},
$$

i.e. $H^{1,0}(X, \mathbb{C})$ consists of holomorphic 1 -forms on $X$. Denoting $h^{1,0}(X):=$ $\operatorname{dim}_{\mathbb{C}} H^{1,0}(X, \mathbb{C})$ we have the following trivial:

Observation 3.8. If $X$ is complex parallelisabilisable, then $h^{1,0}(X)=n$.

Proof. By the complex parallelisability hypothesis on $X$, the rank- $n$ analytic sheaf $\Omega_{X}^{1}$ is trivial, hence it is generated by $n$ holomorphic 1 -forms $\varphi_{1}, \ldots, \varphi_{n} \in H^{1,0}(X, \mathbb{C})$ that are linearly independent at every point of $X$. In particular, $\left\{\varphi_{1}, \ldots, \varphi_{n}\right\}$ is a basis of $H^{1,0}(X, \mathbb{C}) \simeq H^{0}\left(X, \Omega_{X}^{1}\right)$.

Suppose now that $X$ is compact complex parallelisable. Let $\theta_{1}, \ldots, \theta_{n} \in$ $H^{0}\left(X, T^{1,0} X\right)$ be $n$ holomorphic vector fields that are linearly independent at every point of $X$, chosen to be dual to the holomorphic $(1,0)$-forms $\varphi_{1}, \ldots, \varphi_{n} \in$ $H^{1,0}(X, \mathbb{C})$ considered in the above proof. For every smooth function $g: X \rightarrow \mathbb{C}$, we have

$$
\partial g=\sum_{\lambda=1}^{n}\left(\theta_{\lambda} g\right) \varphi_{\lambda}, \quad \bar{\partial} g=\sum_{\lambda=1}^{n}\left(\bar{\theta}_{\lambda} g\right) \bar{\varphi}_{\lambda},
$$

i.e. the familiar formalism induced by local holomorphic coordinates finds a global analogue on a compact complex parallelisable manifold in a formalism where $\theta_{\lambda}$ replaces $\partial / \partial z_{\lambda}$ and $\varphi_{\lambda}$ replaces $d z_{\lambda}$. Thus any $(0,1)$-form $\varphi$ on $X$ has a unique decomposition

$$
\varphi=\sum_{\lambda=1}^{n} f_{\lambda} \bar{\varphi}_{\lambda}
$$

with $f_{1}, \ldots, f_{n}: X \rightarrow \mathbb{C}$ functions on $X$. Thus there is an implicit $L^{2}$ inner product on $C^{\infty}\left(X, \Lambda^{0,1} T^{\star} X\right)$ defined as follows (no Hermitian metric is needed on $X)$ : for any $\varphi=\sum_{\lambda=1}^{n} f_{\lambda} \bar{\varphi}_{\lambda}, \psi=\sum_{\lambda=1}^{n} g_{\lambda} \bar{\varphi}_{\lambda} \in C^{\infty}\left(X, \Lambda^{0,1} T^{\star} X\right)$, set

$$
\langle\langle\varphi, \psi\rangle\rangle:=\int_{X}\left(\sum_{\lambda=1}^{n} f_{\lambda} \bar{g}_{\lambda}\right) i^{n^{2}} \varphi_{1} \wedge \cdots \wedge \varphi_{n} \wedge \bar{\varphi}_{1} \wedge \cdots \wedge \bar{\varphi}_{n} .
$$


It is clear that $d V:=i^{n^{2}} \varphi_{1} \wedge \cdots \wedge \varphi_{n} \wedge \bar{\varphi}_{1} \wedge \cdots \wedge \bar{\varphi}_{n}>0$ is a global volume form on $X$ and that the above $L^{2}$ inner product is independent of the choices made. We can define the formal adjoint $\bar{\partial}^{\star}$ of $\bar{\partial}$ with respect to this $L^{2}$ inner product in the usual way: for any smooth $(0,1)$-form $\varphi$, define $\bar{\partial}^{\star} \varphi$ to be the unique smooth function on $X$ satisfying

$$
\left\langle\left\langle\bar{\partial}^{\star} \varphi, g\right\rangle\right\rangle=\langle\langle\varphi, \bar{\partial} g\rangle\rangle
$$

for any smooth function $g$ on $X$. A trivial calculation using Stokes' theorem gives

$$
\bar{\partial}^{\star} \varphi=-\sum_{\lambda=1}^{n} \theta_{\lambda} f_{\lambda}
$$

for any smooth $(0,1)$-form $\varphi=\sum_{\lambda=1}^{n} f_{\lambda} \bar{\varphi}_{\lambda}$ on $X$. Thus we see that

$$
\bar{\partial}^{\star} \bar{\varphi}_{v}=0, \quad v=1, \ldots, n,
$$

because $\bar{\varphi}_{\nu}=\sum_{\lambda=1}^{n} \delta_{\nu \lambda} \bar{\varphi}_{\lambda}$ and $\theta_{\lambda} \delta_{\nu \lambda}=0$ (since the $\delta_{\nu \lambda}$ are constants).

Now denote by $r \in\{0,1, \ldots, n\}$ the number of $d$-closed forms among $\varphi_{1}, \ldots, \varphi_{n}$. After a possible reordering, we can suppose that $\varphi_{1}, \ldots, \varphi_{r}$ are $d$-closed and $\varphi_{r+1}, \ldots, \varphi_{n}$ are not $d$-closed. Then we have

$$
\partial \varphi_{1}=\cdots=\partial \varphi_{r}=0 \quad \text { or equivalently } \quad \bar{\partial} \bar{\varphi}_{1}=\cdots=\bar{\partial} \bar{\varphi}_{r}=0 .
$$

Thus the $\bar{\partial}$-closed $(0,1)$-forms $\bar{\varphi}_{1}, \ldots, \bar{\varphi}_{r}$ define Dolbeault $(0,1)$-cohomology classes in $H^{0,1}(X, \mathbb{C})$.

We can define the $\bar{\partial}$-Laplacian on forms of $X$ in the usual way:

$$
\Delta^{\prime \prime}:=\bar{\partial} \bar{\partial}^{\star}+\bar{\partial}^{\star} \bar{\partial}
$$

The corresponding harmonic space of $(0,1)$-forms $\mathcal{H}_{\Delta^{\prime \prime}}^{0,1}(X, \mathbb{C}):=\operatorname{ker} \Delta^{\prime \prime}=\operatorname{ker} \bar{\partial} \cap$ ker $\bar{\partial}^{\star}$ satisfies the Hodge isomorphism $\mathcal{H}_{\Delta^{\prime \prime}}^{0,1}(X, \mathbb{C}) \simeq H^{0,1}(X, \mathbb{C})$. Notice that (3.11) and (3.12) give

$$
\Delta^{\prime \prime} \bar{\varphi}_{\nu}=0, \quad v=1, \ldots, r
$$

i.e. the forms $\bar{\varphi}_{1}, \ldots, \bar{\varphi}_{r}$ are $\Delta^{\prime \prime}$-harmonic. On the other hand, $\bar{\varphi}_{r+1}, \ldots, \bar{\varphi}_{n}$ are not $\Delta^{\prime \prime}$-harmonic. Thus the number $r$ of linearly independent $d$-closed holomorphic 1-forms of $X$ (independent of the choice of $\varphi_{1}, \ldots, \varphi_{n}$ ) satisfies:

$$
r \leq h^{0,1}(X)
$$

Suppose now that the compact complex parallelisable $X$ is nilpotent. 
Fact 3.9 (see e.g. [37] or [13, page 5405-5406]). If $X$ is a compact complex parallelisable nilmanifold, then the holomorphic 1 -forms $\varphi_{1}, \ldots, \varphi_{n}$ that form a basis of $H^{1,0}(X, \mathbb{C})$ can be chosen such that

$$
d \varphi_{\mu}=\sum_{1 \leq \lambda<\nu \leq n} c_{\mu \lambda \nu} \varphi_{\lambda} \wedge \varphi_{\nu}, \quad 1 \leq \mu \leq n,
$$

with constant coefficients $c_{\mu \lambda \nu} \in \mathbb{C}$ satisfying

$$
c_{\mu \lambda \nu}=0 \text { whenever } \mu \leq \lambda \text { or } \mu \leq \nu .
$$

Taking this standard fact (which in [37] follows from the existence of a Chevalley decomposition of the nilpotent Lie algebra $\mathfrak{g}$ ) for granted, we now spell out the details of the proof of the following result of Kodaira along the lines of [37, Theorem 3, page 100]:

Theorem 3.10 (Kodaira). If $X$ is a compact complex parallelisable nilmanifold then $h^{0,1}(X)=r$. Moreover, the $\Delta^{\prime \prime}$-harmonic $(0,1)$-forms $\bar{\varphi}_{1}, \ldots, \bar{\varphi}_{r}$ form a basis of the harmonic space $\mathcal{H}_{\Delta^{\prime \prime}}^{0,1}(X, \mathbb{C})$. Equivalently, the Dolbeault $(0,1)$-cohomology classes $\left\{\bar{\varphi}_{1}\right\}, \ldots,\left\{\bar{\varphi}_{r}\right\}$ form a basis of $H^{0,1}(X, \mathbb{C})$.

Proof. The only thing that needs proving is that the linearly independent forms $\bar{\varphi}_{1}, \ldots, \bar{\varphi}_{r} \in \mathcal{H}_{\Delta^{\prime \prime}}^{0,1}(X, \mathbb{C})$ generate $\mathcal{H}_{\Delta^{\prime \prime}}^{0,1}(X, \mathbb{C})$. Pick an arbitrary $C^{\infty}(0,1)$-form $\varphi$ on $X$ and write

$$
\varphi=\sum_{\lambda=1}^{n} f_{\lambda} \bar{\varphi}_{\lambda}
$$

with $C^{\infty}$ functions $f_{1}, \ldots, f_{n}$ on $X$. Using formula (3.8) for $\bar{\partial}$ and the obvious identities $\bar{\partial} \bar{\varphi}_{\lambda}=d \bar{\varphi}_{\lambda}, \lambda=1, \ldots, n$, due to $\varphi_{\lambda}$ being holomorphic, we get:

$$
\begin{aligned}
\bar{\partial} \varphi & =\sum_{\lambda, \nu=1}^{n}\left(\bar{\theta}_{\nu} f_{\lambda}\right) \bar{\varphi}_{\nu} \wedge \bar{\varphi}_{\lambda}+\sum_{\mu=1}^{n} f_{\mu} d \bar{\varphi}_{\mu} \\
& =\sum_{\lambda, \nu=1}^{n}\left(\bar{\theta}_{\nu} f_{\lambda}\right) \bar{\varphi}_{\nu} \wedge \bar{\varphi}_{\lambda}+\sum_{\mu=1}^{n} f_{\mu} \sum_{1 \leq \nu<\lambda \leq n} \overline{c_{\mu \nu \lambda}} \bar{\varphi}_{\nu} \wedge \bar{\varphi}_{\lambda} \\
& =\sum_{1 \leq \nu<\lambda \leq n}\left(\bar{\theta}_{\nu} f_{\lambda}-\bar{\theta}_{\lambda} f_{\nu}+\sum_{\mu=1}^{n} \overline{c_{\mu \nu \lambda}} f_{\mu}\right) \bar{\varphi}_{\nu} \wedge \bar{\varphi}_{\lambda},
\end{aligned}
$$

where the second line above follows from the conjugate of (3.15). 
Now $\varphi$ is $\Delta^{\prime \prime}$-harmonic if and only if

(i) $\bar{\partial} \varphi=0 \Longleftrightarrow \bar{\theta}_{\nu} f_{\lambda}-\bar{\theta}_{\lambda} f_{\nu}+\sum_{\mu=1}^{n} \bar{c}_{\mu \nu \lambda} f_{\mu}=0 \quad$ for $1 \leq v<\lambda \leq n$ (cf. (3.16)); and

(ii) $\bar{\partial}^{\star} \varphi=0 \Longleftrightarrow \sum_{\lambda=1}^{n} \theta_{\lambda} f_{\lambda}=0 \quad(c f .(3.10))$.

Suppose that $\varphi$ is $\Delta^{\prime \prime}$-harmonic. Then the above (i) reads:

$$
\bar{\theta}_{\lambda} f_{\nu}=\sum_{\mu=1}^{n} \overline{c_{\mu \nu \lambda}} f_{\mu}+\bar{\theta}_{\nu} f_{\lambda}, \quad 1 \leq v<\lambda \leq n .
$$

Summing over $\lambda=1, \ldots, n$ and using formula (3.8) for $\bar{\partial}$, we get

$$
\bar{\partial} f_{\nu}=\sum_{\lambda=1}^{n}\left(\bar{\theta}_{\lambda} f_{\nu}\right) \bar{\varphi}_{\lambda}=\sum_{\lambda, \mu=1}^{n} \overline{c_{\mu \nu \lambda}} f_{\mu} \bar{\varphi}_{\lambda}+\sum_{\lambda=1}^{n}\left(\bar{\theta}_{\nu} f_{\lambda}\right) \bar{\varphi}_{\lambda}, \quad v=1, \ldots, n,
$$

with the understanding that $c_{\mu \nu \lambda}=0$ if $v \geq \lambda$. Now $\Delta^{\prime \prime} f_{v}=\bar{\partial} \star \bar{\partial} f_{v}$ since $f_{v}$ is a function. Taking $\bar{\partial}^{\star}$ on either side above and using formula (3.10) for $\bar{\partial}^{\star}$, we get

$$
\begin{aligned}
\Delta^{\prime \prime} f_{\nu} & =-\sum_{\lambda, \mu=1}^{n} \theta_{\lambda}\left(\overline{c_{\mu \nu \lambda}} f_{\mu}\right)-\sum_{\lambda=1}^{n} \theta_{\lambda}\left(\bar{\theta}_{\nu} f_{\lambda}\right) \\
& =-\sum_{\lambda, \mu=1}^{n} \overline{c_{\mu \nu \lambda}} \theta_{\lambda} f_{\mu}, \quad \text { for all } v=1, \ldots, n,
\end{aligned}
$$

because $\theta_{\lambda}\left(\overline{c_{\mu \nu \lambda}} f_{\mu}\right)=\overline{c_{\mu \nu \lambda}} \theta_{\lambda} f_{\mu}$ due to $\overline{c_{\mu \nu \lambda}}$ being constant, while $\sum_{\lambda=1}^{n} \theta_{\lambda} f_{\lambda}=0$ due to $\varphi$ being $\Delta^{\prime \prime}$-harmonic (cf. (ii) or (3.10)).

Taking now $\nu=n$ in (3.18), we get $\Delta^{\prime \prime} f_{n}=0$ since $c_{\mu n \lambda}=0$ for all $\mu, \lambda$ by Fact 3.9 and the obvious inequality $\mu \leq v=n$. Thus the compactness of $X$ and the ellipticity of $\Delta^{\prime \prime}$ yield

$$
f_{n} \text { is constant on } X \text { if } \Delta^{\prime \prime} \varphi=0 \text {. }
$$

Taking now $v=n-1$ in (3.18) and using the fact that $\theta_{\lambda} f_{n}=0$ for all $\lambda$ (since $f_{n}$ is constant by (3.18)), we get

$$
\Delta^{\prime \prime} f_{n-1}=-\sum_{\lambda=1}^{n}\left(\sum_{\mu=1}^{n-1} \overline{c_{\mu n-1 \lambda}} \theta_{\lambda} f_{\mu}\right)=0 \text { on } X,
$$

since $c_{\mu n-1 \lambda}=0$ for all $\mu=1, \ldots, n-1$ and $\lambda=1, \ldots, n$ by Fact 3.9 and the obvious inequality $\mu \leq v=n-1$. Hence we get

$$
f_{n-1} \text { is constant on } X \text { if } \Delta^{\prime \prime} \varphi=0 \text {. }
$$


We can now continue by decreasing induction on $v$. Taking $v=n-2$ in (3.18) and using the fact that $\theta_{\lambda} f_{n}=\theta_{\lambda} f_{n-1}=0$ for all $\lambda$ (since $f_{n}$ is constant by (3.18) and $f_{n-1}$ is constant by (3.19)), we get

$$
\Delta^{\prime \prime} f_{n-2}=-\sum_{\lambda=1}^{n}\left(\sum_{\mu=1}^{n-2} \overline{c_{\mu n-2 \lambda}} \theta_{\lambda} f_{\mu}\right)=0 \quad \text { on } X,
$$

since $c_{\mu n-2 \lambda}=0$ for all $\mu=1, \ldots, n-2$ and $\lambda=1, \ldots, n$ by Fact 3.9 and the obvious inequality $\mu \leq v=n-2$. Hence we get

$$
f_{n-2} \text { is constant on } X \text { if } \Delta^{\prime \prime} \varphi=0 .
$$

Running a decreasing induction on $v$, we get

$$
f_{v}:=C_{v} \quad \text { is constant on } X \text { for all } v=1, \ldots, n \text { if } \Delta^{\prime \prime} \varphi=0 .
$$

We conclude that whenever $\Delta^{\prime \prime} \varphi=0$ we have

$$
\varphi=\sum_{\nu=1}^{n} C_{\nu} \bar{\varphi}_{\nu} \quad \text { with } C_{\nu} \text { constant for all } v=1, \ldots, n .
$$

On the other hand, since $\Delta^{\prime \prime} \varphi=0$, we must have $\bar{\partial} \varphi=0$ which amounts to $\sum_{\nu=1}^{n} C_{\nu} \bar{\partial} \bar{\varphi}_{\nu}=0$. However, we know that $\bar{\partial} \bar{\varphi}_{\nu}=0$ for all $\nu \in\{1, \ldots, r\}(c f$. (3.12)), hence $\sum_{\nu=r+1}^{n} C_{\nu} \bar{\partial} \bar{\varphi}_{\nu}=0$. Now the forms

$$
\bar{\partial} \bar{\varphi}_{\nu}=d \bar{\varphi}_{\nu}=\sum_{\lambda, \mu}{\overline{c_{\nu \lambda \mu}}}_{\bar{\varphi}_{\lambda}} \wedge \bar{\varphi}_{\mu}, \quad v=1, \ldots, n,
$$

are linearly independent because $\bar{\varphi}_{1}, \ldots, \bar{\varphi}_{n}$ are linearly independent at every point of $X$. Hence $C_{v}=0$ for all $v=r+1, \ldots, n$. We get

$$
\varphi=\sum_{\nu=1}^{r} C_{\nu} \bar{\varphi}_{\nu} \quad \text { with } C_{\nu} \text { constant for all } v=1, \ldots, r .
$$

Since $\varphi$ has been chosen arbitrary in $\mathcal{H}_{\Delta^{\prime \prime}}^{0,1}(X, \mathbb{C})$, we have proved that the linearly independent forms $\bar{\varphi}_{1}, \ldots, \bar{\varphi}_{r} \in \mathcal{H}_{\Delta^{\prime \prime}}^{0,1}(X, \mathbb{C})$ generate $\mathcal{H}_{\Delta^{\prime \prime}}^{0,1}(X, \mathbb{C})$. The proof of Kodaira's theorem 3.10 is complete.

When applying Observation 3.8 and Kodaira's Theorem 3.10 to the Iwasawa manifold (a compact complex parallelisable nilmanifold of dimension $n=3$ having $r=2$ ), we get the following classical fact: 
Observation 3.11. For the Iwasawa manifold, we have

$$
h^{1,0}=3 \quad \text { and } \quad h^{0,1}=2 .
$$

Since, on the other hand, the first Betti number is $b_{1}=4$ (see e.g. (3.4)), we see that $b_{1}<h^{1,0}+h^{0,1}$. Thus the Frölicher spectral sequence of the Iwasawa manifold does not degenerate at $E_{1}{ }^{9}$. In particular, the $\partial \bar{\partial}$-lemma does not hold on the Iwasawa manifold.

On the other hand, we have seen in Corollary 3.6 that the Iwasawa manifold is balanced, hence sG. Combined with Observation 3.11, this fact proves part $(a)$ of Theorem 1.11. The same statement will be proved again in section 4 (see comments after Gauduchon's Theorem 4.9) by a twistor space featuring as the central fibre in the Eastwood-Singer family [16].

(b) Now suppose that $X$ is the Iwasawa manifold. In particular, $X$ is a compact complex parallelisable nilmanifold of complex dimension 3 . Let $\varphi_{1}=d z_{1}, \varphi_{2}=$ $d z_{2}, \varphi_{3}=d z_{3}-z_{1} d z_{2}$ be the holomrophic 1-forms on $X$ defined in (3.2); they are linearly independent at every point of $X$. Since $\varphi_{1}$ and $\varphi_{2}$ are $d$-closed while $\varphi_{3}$ is not $d$-closed, $r=2$ for the Iwasawa manifold. By Kodaira's Theorem 3.10, the $\mathbb{C}$-vector space $H^{0,1}(X, \mathbb{C})$ has complex dimension 2 and is spanned by the Dolbeault cohomology classes $\left\{\bar{\varphi}_{1}\right\}$ and $\left\{\bar{\varphi}_{2}\right\}$. Let $\theta_{1}, \theta_{2}, \theta_{3} \in H^{0}\left(X, \Omega_{X}^{1}\right)$ be the holomorphic vector fields dual to $\varphi_{1}, \varphi_{2}, \varphi_{3}$. They are given by

$$
\theta_{1}=\frac{\partial}{\partial z_{1}}, \theta_{2}=\frac{\partial}{\partial z_{2}}+z_{1} \frac{\partial}{\partial z_{3}}, \theta_{3}=\frac{\partial}{\partial z_{3}}
$$

and satisfy the relations

$$
\left[\theta_{1}, \theta_{2}\right]=-\left[\theta_{2}, \theta_{1}\right]=\theta_{3}, \quad\left[\theta_{1}, \theta_{3}\right]=\left[\theta_{2}, \theta_{3}\right]=0,
$$

i.e. $\left[\theta_{i}, \theta_{j}\right]=0$ whenever $\{i, j\} \neq\{1,2\}$.

Since the holomorphic tangent bundle $T^{1,0} X$ is trivial and spanned by $\theta_{1}, \theta_{2}, \theta_{3}$, the cohomology group $H^{0,1}\left(X, T^{1,0} X\right)$ of $T^{1,0} X$-valued $(0,1)$-forms on $X$ is a $\mathbb{C}$ vector space of dimension 6 spanned by the classes of $\theta_{i} \bar{\varphi}_{\lambda}$ :

$$
H^{0,1}\left(X, T^{1,0} X\right)=\bigoplus_{1 \leq i \leq 3,1 \leq \lambda \leq 2} \mathbb{C}\left\{\theta_{i} \bar{\varphi}_{\lambda}\right\}, \quad \operatorname{dim}_{\mathbb{C}} H^{0,1}\left(X, T^{1,0} X\right)=6 .
$$

This will be seen to imply that the Kuranishi family of the Iwasawa manifold is a 6 -parameter family.

(c) We now briefly recall a few basic facts in the deformation theory of compact complex manifolds in order to fix the notation. The standard reference is Kodaira's

${ }^{9}$ Historically, the Iwasawa manifold was the first example exhibited of a compact complex manifold with this property. It is even known to have $E_{1} \neq E_{2}=E_{\infty}$ in its Frölicher spectral sequence. 
book [28]. Given a compact complex manifold $X$ of complex dimension $n$, let $\bar{\partial}$ be the Cauchy-Riemann operator representing the complex structure $J=J_{0}$ of $X$ and let $z_{1}, \ldots, z_{n}$ be local holomorphic coordinates about an arbitrary point on $X$. A deformation $X_{t}$ of the complex structure of $X$ is represented by a vector $(0,1)$-form

$$
\psi(t) \in C^{\infty}\left(X, \Lambda^{0,1} T^{\star} X \otimes T^{1,0} X\right)
$$

in the following sense: the Cauchy-Riemann operator $\bar{\partial}_{t}$ representing the (almost) complex structure $J_{t}$ of $X_{t}$ is defined by

$$
\bar{\partial}_{t}:=\bar{\partial}-\psi(t)
$$

Equivalently, a locally defined $C^{\infty}$ function $f$ on $X$ satisfies the equivalence:

$$
f \text { is } J_{t} \text {-holomorphic if and only if }(\bar{\partial}-\psi(t)) f=0 \text {. }
$$

The integrability condition reads:

$$
\begin{aligned}
J_{t} \text { is integrable } \text { if and only if } & \bar{\partial} \psi(t)=\frac{1}{2}[\psi(t), \psi(t)] \\
\text { if and only if } & \text { the system of n PDE's } \bar{\partial}_{t} f=0 \text { has } \\
& n \text { linearly independent } C^{\infty} \text { solutions in } \\
& \text { a neighbourhood of any point of } X .
\end{aligned}
$$

Recall furthermore that the Kodaira-Spencer map of a holomorphic family $\pi$ : $\mathcal{X} \longrightarrow B \subset \mathbb{C}^{m}$ at $t=0$ (having supposed that $0 \in B$ ) is

$$
\rho_{0}: T_{0} B \longrightarrow H^{0,1}\left(X, T^{1,0} X\right), \quad \frac{\partial}{\partial t} \mapsto-\frac{\partial \psi(t)}{\partial t}_{\mid t=0}:={\frac{\partial X_{t}}{\partial t}}_{\mid t=0} .
$$

Thus ${\frac{\partial X_{t}}{\partial t}}_{\mid t=0}$ denotes the infinitesimal deformation of $X$ at $t=0$. Recall now the fundamental Kuranishi Theorem of Existence:

Theorem 3.12 (Kuranishi [30]). Given any compact complex manifold $X$, there exists a complete holomorphic family

$$
\pi: \mathcal{X} \longrightarrow B \subset \Delta_{\varepsilon}:=\left\{t \in \mathbb{C}^{m} ;|t|<\varepsilon\right\}
$$

such that $X_{0}=X$, for some small $\varepsilon>0$ and some analytic subset $B$ of the ball $\Delta_{\varepsilon}$, where $m:=\operatorname{dim}_{\mathbb{C}} H^{0,1}\left(X, T^{1,0} X\right)$.

Complete means that the Kodaira-Spencer map $\rho_{0}: T_{0} B \longrightarrow H^{0,1}\left(X, T^{1,0} X\right)$ is surjective. This family is called the Kuranishi family of $X$. The base $B$ of the family may have singularities and arises as

$$
B=\left\{t \in \Delta_{\varepsilon} ; f_{1}(t)=\cdots=f_{l}(t)=0\right\},
$$


where $l:=\operatorname{dim}_{\mathbb{C}} H^{0,2}\left(X, T^{1,0} X\right)$ and $f_{1}, \ldots, f_{l}: \Delta_{\varepsilon} \rightarrow \mathbb{C}$ are holomorphic functions. In the special case of a manifold $X$ satisfying $H^{0,2}\left(X, T^{1,0} X\right)=0$, the base $B$ is smooth and Kuranishi's theorem reduces to the earlier Kodaira-NirenbergSpencer theorem of existence.

The construction of the Kuranishi family of a given $X$ amounts to the construction of a family of vector $(0,1)$-forms $\psi(t) \in C^{\infty}\left(X, \Lambda^{0,1} T^{\star} X \otimes T^{1,0} X\right)$ satisfying the integrability condition (3.25) for $t=\left(t_{1}, \ldots, t_{m}\right)$ in the largest possible subset of some $\Delta_{\varepsilon} \subset \mathbb{C}^{m}$. Given an arbitrary basis $\left\{\beta_{1}, \ldots, \beta_{m}\right\}$ of $H^{0,1}\left(X, T^{1,0} X\right)$, set

$$
\psi_{1}(t):=t_{1} \beta_{1}+\ldots t_{m} \beta_{m} \in H^{0,1}\left(X, T^{1,0} X\right) .
$$

Identifying $H^{0,1}\left(X, T^{1,0} X\right)$ with $\mathcal{H}_{\Delta^{\prime \prime}}^{0,1}\left(X, T^{1,0} X\right)$ by the Hodge isomorphism, we see that $\Delta^{\prime \prime} \psi_{1}(t)=0$ (i.e. $\psi_{1}(t)$ is $\Delta^{\prime \prime}$-harmonic).

Since $\psi_{1}$ need not satisfy the integrability condition (3.25), we search for a power series

$$
\psi(t)=\psi_{1}(t)+\sum_{\nu=2}^{+\infty} \psi_{\nu}(t)
$$

where $\psi_{\nu}(t)=\sum_{\nu_{1}+\cdots+v_{m}=\nu} \psi_{\nu_{1} \ldots v_{m}} t_{1}^{\nu_{1}} \ldots t_{m}^{\nu_{m}}$ is a homogeneous polynomial of degree $v$ in $t_{1}, \ldots, t_{m}$ whose coefficients $\psi_{v_{1} \ldots v_{m}} \in C^{\infty}\left(X, \Lambda^{0,1} T^{\star} X \otimes T^{1,0} X\right)$ will be determined such that the following two conditions are fulfilled:

- The power series defining $\psi(t)$ converges and its sum is $C^{\infty}$ on $X \times \Delta_{\varepsilon}$ for some small $\varepsilon>0$;

Kuranishi's proof achieves convergence in a Hölder norm ||$_{k, \alpha}$ for all $k \geq 2$ and all $t \in \Delta_{\varepsilon}$ provided that $\varepsilon>0$ is small enough.

- The integrability condition $\bar{\partial} \psi(t)=\frac{1}{2}[\psi(t), \psi(t)]$ holds (cf. (3.25)).

To fulfill the integrability condition (3.25), it suffices to ensure that the vector $(0,2)$-form $[\psi(t), \psi(t)] \in C^{\infty}\left(X, \Lambda^{0,2} T^{\star} X \otimes T^{1,0} X\right)$ is $\bar{\partial}$-exact and that $\psi(t)-$ $\psi_{1}(t)$ is the minimal $L^{2}$-norm solution of equation $\bar{\partial} u=\frac{1}{2}[\psi(t), \psi(t)]$ (recall that $\left.\bar{\partial} \psi_{1}(t)=0\right)$. Minimality of the solution's $L^{2}$-norm translates to

$$
\psi(t)=\psi_{1}(t)+\frac{1}{2} \bar{\partial}^{\star} \Delta^{\prime \prime}-1[\psi(t), \psi(t)],
$$

a formula that is easily seen to be equivalent to

$$
\psi_{\nu}(t)=\frac{1}{2} \sum_{\mu=1}^{\nu-1} \bar{\partial}^{\star} \Delta^{\prime \prime}-1\left[\psi_{\mu}(t), \psi_{\nu-\mu}(t)\right] \text { for all } v \geq 2 .
$$

This means that $\psi_{\nu}(t)$ is the minimal $L^{2}$-norm solution of the equation $\bar{\partial} u=v_{\nu}$, where $v_{\nu}$ is the projection of $1 / 2 \sum_{1 \leq \mu \leq \nu-1}\left[\psi_{\mu}(t), \psi_{\nu-\mu}(t)\right]$ onto $\operatorname{Im} \bar{\partial}$. In particular, $\psi_{v}(t) \in \operatorname{Im} \bar{\partial}^{\star}$ for all $v \geq 2$. 
Identities (3.29) allow one to construct $\psi_{v}(t), v \geq 2$, inductively from $\psi_{1}(t)$ defined in (3.27). Convergence in Hölder norm ||$_{k, \alpha}$ of the resulting series (3.28) follows from a priori estimates on the Laplacian $\Delta^{\prime \prime}$, while the integrability condition (3.25) for the sum $\psi(t)$ of this series is seen to be equivalent to

$$
H[\psi(t), \psi(t)]=0,
$$

where $H: C^{\infty}\left(X, \Lambda^{0,2} T^{\star} X \otimes T^{1,0} X\right) \rightarrow \mathcal{H}_{\Delta^{\prime \prime}}^{0,2}\left(X, T^{1,0} X\right)$ is the harmonic projector. Condition (3.30) requires $[\psi(t), \psi(t)]$ to have no harmonic component which, for a $\bar{\partial}$-closed form, is equivalent to $\bar{\partial}$-exactness (precisely what is needed in view of (3.25)). If $\left\{\gamma_{1}, \ldots, \gamma_{l}\right\}$ is any orthonormal basis of $\mathcal{H}_{\Delta^{\prime \prime}}^{0,2}\left(X, T^{1,0} X\right)$, then

$$
H[\psi(t), \psi(t)]=\sum_{k=1}^{l}\left\langle[\psi(t), \psi(t)], \gamma_{k}\right\rangle \gamma_{k}, \quad t \in \Delta_{\varepsilon},
$$

and we see that the vanishing condition (3.30) is equivalent to $f_{1}(t)=\cdots=$ $f_{l}(t)=0$, where $f_{k}(t):=\left\langle[\psi(t), \psi(t)], \gamma_{k}\right\rangle$ for all $k=1, \ldots, l$ and $t \in \Delta_{\varepsilon}$. Thus the integrability condition (3.25) is satisfied for $t \in B$, where

$$
B:=\left\{t \in \Delta_{\varepsilon} ; f_{1}(t)=\cdots=f_{l}(t)=0\right\} \subset \Delta_{\varepsilon}
$$

is analytic.

(d) Nakamura's calculation of the Kuranishi family of the Iwasawa manifold

Given a compact complex manifold $X$ of dimension $n$, for any vector $(0,1)$ forms $\psi, \tau \in C^{\infty}\left(X, \Lambda^{0,1} T^{\star} X \otimes T^{1,0} X\right)$ written locally as

$$
\psi=\sum_{\alpha=1}^{n} \psi^{\alpha} \frac{\partial}{\partial z_{\alpha}}, \quad \tau=\sum_{\beta=1}^{n} \tau^{\beta} \frac{\partial}{\partial z_{\beta}}, \quad \psi^{\alpha}, \tau^{\beta} \in C^{\infty}\left(X, T^{1,0} X\right),
$$

Kuranishi defines in general

$$
[\psi, \tau]:=\sum_{\alpha, \beta=1}^{n}\left(\psi^{\alpha} \wedge \frac{\partial \tau^{\beta}}{\partial z_{\alpha}}+\tau^{\alpha} \wedge \frac{\partial \psi^{\beta}}{\partial z_{\alpha}}\right) \frac{\partial}{\partial z_{\beta}} .
$$

Now fix $X=\mathbb{C}^{3} / \Gamma$ to be the Iwasawa manifold. Then $n=3$ and we get:

$$
\left[\theta_{i} \bar{\varphi}_{\lambda}, \theta_{k} \bar{\varphi}_{\nu}\right]=\left[\theta_{i}, \theta_{k}\right] \bar{\varphi}_{\lambda} \wedge \bar{\varphi}_{\nu}, \quad i, k, \lambda, v=1,2,3,
$$

with $\left[\theta_{i}, \theta_{k}\right]$ given in (3.23).

We have seen in (3.24) that the classes $\left\{\theta_{i} \overline{\varphi_{\lambda}}\right\}$, with $1 \leq i \leq 3,1 \leq \lambda \leq 2$, form a basis of $H^{0,1}\left(X, T^{1,0} X\right)$. Consequently the Kuranishi family of $X$ can be described by 6 parameters $t=\left(t_{i \lambda}\right)_{1 \leq i \leq 3,1 \leq \lambda \leq 2}$. By (3.13), the $T^{1,0} X$-valued $(0,1)$-forms $\theta_{i} \overline{\varphi_{\lambda}}$ are $\Delta^{\prime \prime}$-harmonic when $1 \leq \lambda \leq 2$. In order to construct the 
vector $(0,1)$-forms $\psi(t) \in C^{\infty}\left(X, \Lambda^{0,1} T^{\star} X \otimes T^{1,0} X\right)$ that describe the Kuranishi family of $X=\mathbb{C}^{3} / \Gamma$, formula (3.27) prescribes to start off by setting

$$
\psi_{1}(t):=\sum_{i=1}^{3} \sum_{\lambda=1}^{2} t_{i \lambda} \theta_{i} \bar{\varphi}_{\lambda}, \quad t=\left(t_{i \lambda}\right)_{1 \leq i \leq 3,1 \leq \lambda \leq 2}
$$

for which we see that

$$
\frac{1}{2}\left[\psi_{1}(t), \psi_{1}(t)\right]=\frac{1}{2} \sum_{i, j=1,2,3} \sum_{\lambda, \mu=1,2} t_{i \lambda} t_{j \mu}\left[\theta_{i}, \theta_{j}\right] \bar{\varphi}_{\lambda} \wedge \bar{\varphi}_{\mu} .
$$

By (3.23), this translates to

$$
\begin{aligned}
\frac{1}{2}\left[\psi_{1}(t), \psi_{1}(t)\right]=\frac{1}{2}\left(t_{11} t_{22} \theta_{3} \bar{\varphi}_{1} \wedge \bar{\varphi}_{2}\right. & +t_{12} t_{21} \theta_{3} \bar{\varphi}_{2} \wedge \bar{\varphi}_{1} \\
& \left.-t_{21} t_{12} \theta_{3} \bar{\varphi}_{1} \wedge \bar{\varphi}_{2}-t_{22} t_{11} \theta_{3} \bar{\varphi}_{2} \wedge \bar{\varphi}_{1}\right) .
\end{aligned}
$$

Since $\bar{\varphi}_{1} \wedge \bar{\varphi}_{2}=-\bar{\varphi}_{2} \wedge \bar{\varphi}_{1}$, we get

$$
\frac{1}{2}\left[\psi_{1}(t), \psi_{1}(t)\right]=\left(t_{11} t_{22}-t_{12} t_{21}\right) \theta_{3} \bar{\varphi}_{1} \wedge \bar{\varphi}_{2} .
$$

On the other hand, for the choice (3.33) we see that

$$
\bar{\partial} \psi_{1}(t)=d \psi_{1}(t)=\sum_{i=1}^{3} \sum_{\lambda=1}^{2} t_{i \lambda} \theta_{i} d \bar{\varphi}_{\lambda}=0
$$

since $d \bar{\varphi}_{1}=d \bar{\varphi}_{2}=0$. Now setting

$$
\psi_{2}(t):=-\left(t_{11} t_{22}-t_{12} t_{21}\right) \theta_{3} \bar{\varphi}_{3},
$$

and using (3.3) and (3.34), we find

$$
\begin{aligned}
\bar{\partial} \psi_{2}(t) & =d \psi_{2}(t)=\left(t_{11} t_{22}-t_{12} t_{21}\right) \theta_{3}\left(-d \bar{\varphi}_{3}\right) \\
& =\left(t_{11} t_{22}-t_{12} t_{21}\right) \theta_{3} \bar{\varphi}_{1} \wedge \bar{\varphi}_{2}=\frac{1}{2}\left[\psi_{1}(t), \psi_{1}(t)\right] .
\end{aligned}
$$

In particular, $\left[\psi_{1}(t), \psi_{1}(t)\right]$ is seen to be $\bar{\partial}$-exact here (although it need not be so in the case of an arbitrary manifold, see comments after (3.29)), but the solution $\psi_{2}(t)$ of equation (3.37) need not be of minimal $L^{2}$-norm (unlike the $\psi_{2}(t)$ defined in the case of a general manifold by formula (3.29) for $v=2$ ). In other words, in the special case of the Iwasawa manifold, a solution $\psi_{2}(t)$ of (3.37) is easily observed and we are spared the application of the general formulae (3.29). This readily yields the desired $\psi(t)$ by setting

$$
\psi(t):=\psi_{1}(t)+\psi_{2}(t)=\sum_{i=1}^{3} \sum_{\lambda=1}^{2} t_{i \lambda} \theta_{i} \bar{\varphi}_{\lambda}-\left(t_{11} t_{22}-t_{12} t_{21}\right) \theta_{3} \bar{\varphi}_{3},
$$


for which we find

$$
\frac{1}{2}[\psi(t), \psi(t)]=\sum_{j,, k=1}^{2} \frac{1}{2}\left[\psi_{j}(t), \psi_{k}(t)\right]=\frac{1}{2}\left[\psi_{1}(t), \psi_{1}(t)\right] .
$$

Indeed, $\left[\psi_{j}(t), \psi_{k}(t)\right]=0$ for all $(i, j) \neq(1,1)$ since these terms involve only brackets of the shape $\left[\theta_{3}, \theta_{i}\right]=0$ and $\left[\theta_{i}, \theta_{3}\right]=0$ which vanish by (3.23).

On the other hand, combining (3.35) and (3.37), we get

$$
\bar{\partial} \psi(t)=\bar{\partial} \psi_{1}(t)+\bar{\partial} \psi_{2}(t)=\bar{\partial} \psi_{2}(t)=\frac{1}{2}\left[\psi_{1}(t), \psi_{1}(t)\right] .
$$

Then (3.39) and (3.40) yield

$$
\bar{\partial} \psi(t)=\frac{1}{2}[\psi(t), \psi(t)],
$$

showing that $\psi(t)$ defined in (3.38) satisfies the integrability condition (3.25).

By Kuranishi's Theorem 3.12, this $T^{1,0} X$-valued $(0,1)$-form $\psi(t)$ defines a locally complete complex analytic family of deformations $X_{t}$ of $X$ depending on 6 effective parameters $t=\left(t_{\underline{i} \lambda}\right)_{1 \leq i \leq 3,1 \leq \lambda \leq 2}$ such that the complex structure of each fibre $X_{t}$ is defined by $\bar{\partial}_{t}:=\bar{\partial}-\bar{\psi}(t)$ and $X_{0}=X=\mathbb{C}^{3} / \Gamma$ is the Iwasawa manifold. It is noteworthy that in the special case of the Iwasawa manifold, the power series (3.28) can be built with only two terms $\left(\psi_{1}(t)\right.$ and $\left.\psi_{2}(t)\right)$ and the above simple calculations show $\psi(t)=\psi_{1}(t)+\psi_{2}(t)$ to satisfy the integrability condition (3.25) for all $t=\left(t_{i \lambda}\right)_{1 \leq i \leq 3,1 \leq \lambda \leq 2} \in \Delta_{\varepsilon} \subset \mathbb{C}^{6}$ if $\varepsilon>0$ is small. With the notation of (3.31), this means that $B=\Delta_{\varepsilon}$.

Nakamura goes on to calculate holomorphic coordinates $\zeta_{1}=\zeta_{1}(t), \zeta_{2}=$ $\zeta_{2}(t), \zeta_{3}=\zeta_{3}(t)$ on $X_{t}$ such that $\zeta_{v}(0)=z_{v}$ for $v=1,2,3$ starting from arbitrary holomorphic coordinates $z_{1}, z_{2}, z_{3}$ given beforehand on the Iwasawa manifold $X_{0}=X=\mathbb{C}^{3} / \Gamma$. Here is the way he proceeds.

We are looking for $C^{\infty}$ functions $\zeta_{v}(t), v=1,2,3$, on $X$ satisfying the holomorphicity condition

$$
\bar{\partial}_{t} \zeta_{v}(t)=0 \Longleftrightarrow \bar{\partial} \zeta_{v}(t)-\psi(t) \zeta_{v}(t)=0, \quad v=1,2,3 .
$$

Given the definition (3.38) of $\psi(t)$ and the formulae (3.22) for $\theta_{1}, \theta_{2}, \theta_{3}$, condition (3.42) reads for $v=1,2,3$ :

$$
\begin{aligned}
\bar{\partial} \zeta_{v} & -\sum_{\lambda=1}^{2} t_{1 \lambda} \frac{\partial \zeta_{v}}{\partial z_{1}} d \bar{z}_{\lambda}-\sum_{\lambda=1}^{2} t_{2 \lambda}\left(\frac{\partial \zeta_{v}}{\partial z_{2}}+z_{1} \frac{\partial \zeta_{v}}{\partial z_{3}}\right) d \bar{z}_{\lambda} \\
& -\sum_{\lambda=1}^{2} t_{3 \lambda} \frac{\partial \zeta_{v}}{\partial z_{3}} d \bar{z}_{\lambda}+\left(t_{11} t_{22}-t_{12} t_{21}\right) \frac{\partial \zeta_{v}}{\partial z_{3}}\left(d \bar{z}_{3}-\bar{z}_{1} d \bar{z}_{2}\right)=0 .
\end{aligned}
$$


For $v=1$, we arrange to have $\frac{\partial \zeta_{1}}{\partial z_{1}}=1$ (in order to get $\zeta_{1}(t)=z_{1}+$ (terms depending only on $\left.\bar{z}_{\lambda}\right)$ ) and $\frac{\partial \zeta_{1}}{\partial z_{2}}=\frac{\partial \zeta_{1}}{\partial z_{3}}=0$. With these choices, condition (3.43) for $v=1$ becomes:

$$
\bar{\partial} \zeta_{1}-\sum_{\lambda=1}^{2} t_{1 \lambda} \bar{\partial} \bar{z}_{\lambda}=0 \Longleftrightarrow \bar{\partial} \zeta_{1}(t)=\bar{\partial}\left(\sum_{\lambda=1}^{2} t_{1 \lambda} \bar{z}_{\lambda}\right) .
$$

Thus we can take

$$
\zeta_{1}(t)=z_{1}+\sum_{\lambda=1}^{2} t_{1 \lambda} \bar{z}_{\lambda}
$$

For $v=2$, we similarly require $\frac{\partial \zeta_{2}}{\partial z_{2}}=1$ and $\frac{\partial \zeta_{2}}{\partial z_{1}}=\frac{\partial \zeta_{2}}{\partial z_{3}}=0$ and condition (3.43) for $v=2$ similarly yields:

$$
\zeta_{2}(t)=z_{2}+\sum_{\lambda=1}^{2} t_{2 \lambda} \bar{z}_{\lambda}
$$

For $v=3$, we require $\frac{\partial \zeta_{3}}{\partial z_{3}}=1, \frac{\partial \zeta_{3}}{\partial z_{2}}=0$ and $\frac{\partial \zeta_{3}}{\partial z_{1}}=\sum_{\lambda=1}^{2} t_{2 \lambda} \bar{z}_{\lambda}$. With these choices, (3.43) for $v=3$ reads

$$
\begin{aligned}
\bar{\partial} \zeta_{3} & -\left(\sum_{\lambda=1}^{2} t_{1 \lambda} d \bar{z}_{\lambda}\right)\left(\sum_{\lambda=1}^{2} t_{2 \lambda} \bar{z}_{\lambda}\right)-z_{1} \sum_{\lambda=1}^{2} t_{2 \lambda} d \bar{z}_{\lambda} \\
& -\sum_{\lambda=1}^{2} t_{3 \lambda} d \bar{z}_{\lambda}+\left(t_{11} t_{22}-t_{12} t_{21}\right)\left(d \bar{z}_{3}-\bar{z}_{1} d \bar{z}_{2}\right)=0 .
\end{aligned}
$$

We thus get

$$
\zeta_{3}(t)=z_{3}+\sum_{\lambda=1}^{2}\left(t_{3 \lambda}+t_{2 \lambda} z_{1}\right) \bar{z}_{\lambda}+A(t, \bar{z})-D(t) \bar{z}_{3},
$$

where we have denoted $A(t, \bar{z}):=\frac{1}{2}\left(t_{11} t_{21} \bar{z}_{1}^{2}+2 t_{11} t_{22} \bar{z}_{1} \bar{z}_{2}+t_{12} t_{22} \bar{z}_{2}^{2}\right)$ and $D(t):=$ $\left(t_{11} t_{22}-t_{12} t_{21}\right)$. We clearly have

$$
d \zeta_{1}(t) \wedge d \zeta_{2}(t) \wedge d \zeta_{3}(t)=C(t) d z_{1} \wedge d z_{2} \wedge d z_{3}
$$

for a constant $C(t)$ depending in a $C^{\infty}$ way on $t$ such that $C(0)=1$. Hence $\zeta_{1}(t), \zeta_{2}(t), \zeta_{3}(t)$ define holomorphic coordinates on $X_{t}$ for all $t=\left(t_{i \lambda}\right)_{1 \leq i \leq 3,1 \leq \lambda \leq 2}$ such that $\sum_{i=1,2,3 ; \lambda=1,2}\left|t_{i \lambda}\right|<\varepsilon$ if $\varepsilon>0$ is small enough. 


\subsection{The example of Alessandrini and Bassanelli proving Theorem 3.3}

In the 6-parameter Kuranishi family $\left(X_{t}\right)_{t \in B}$, with $t=\left(t_{i \lambda}\right)_{1 \leq i \leq 3,1 \leq \lambda \leq 2}$, of the Iwasawa manifold $X_{0}=X=\mathbb{C}^{3} / \Gamma$, Alessandrini and Bassanelli [2] single out the direction corresponding to parameters $t$ such that

$$
t_{12} \neq 0, \quad t_{i j}=0 \quad \text { for all }(i, j) \neq(1,2) .
$$

With this choice of $t$, they have

$$
A(t, \bar{z})=0 \quad \text { and } \quad D(t)=0 .
$$

Thus, setting $t:=t_{12}$, the holomorphic coordinates of $X_{t}$ calculated in (3.44), (3.45) and (3.46) reduce to

$$
\zeta_{1}(t)=z_{1}+t \bar{z}_{2}, \quad \zeta_{2}(t)=z_{2}, \quad \zeta_{3}(t)=z_{3} .
$$

Implicitly $z_{1}=\zeta_{1}(t)-t \bar{\zeta}_{2}(t)$, which yields

$$
\begin{aligned}
& \varphi_{3}(t):=d z_{3}-z_{1} d z_{2}=d \zeta_{3}(t)+\left(t \bar{\zeta}_{2}(t)-\zeta_{1}(t)\right) d \zeta_{2}(t), \\
& \varphi_{2}(t):=d z_{2}=d \zeta_{2}(t), \quad \widetilde{\varphi}_{1}(t):=d z_{1}=d \zeta_{1}(t)-t d \bar{\zeta}_{2}(t) .
\end{aligned}
$$

Set

$$
\varphi_{1}(t):=d \zeta_{1}(t)
$$

The above 1 -forms $\varphi_{1}(t), \varphi_{2}(t), \varphi_{3}(t)$ are all of $J_{t}$-type $(1,0)$ since $\zeta_{1}(t), \zeta_{2}(t)$, $\zeta_{3}(t)$ are holomorphic coordinates for the complex structure $J_{t}$ of $X_{t}$.

Proposition 3.13 (Alessandrini-Bassanelli [2, page 1062]). Let $\left(X_{t}\right)_{t}$ be the Kuranishi family of the Iwasawa manifold $X=X_{0}, t=\left(t_{i \lambda}\right)_{1 \leq i \leq 3,1 \leq \lambda \leq 2}$. Then, for parameters such that $t_{i \lambda}=0$ for all $(i, \lambda) \neq(1,2), X_{t}$ is not balanced for any $t:=t_{12} \neq 0$ satisfying $\left|t_{12}\right|<\varepsilon$ if $\varepsilon>0$ is small enough.

Proof. For the forms defined in (3.49) and (3.50), an immediate calculation shows

$$
d \varphi_{3}(t)=\left(t d \bar{\zeta}_{2}(t)-d \zeta_{1}(t)\right) \wedge d \zeta_{2}(t)=-t \varphi_{2}(t) \wedge \bar{\varphi}_{2}(t)-\varphi_{1}(t) \wedge \varphi_{2}(t) .
$$

Thus the 2-form $d \varphi_{3}(t)$ has two components: $-t \varphi_{2}(t) \wedge \bar{\varphi}_{2}(t)$ is of $J_{t}$-type $(1,1)$, while $-\varphi_{1}(t) \wedge \varphi_{2}(t)$ is of $J_{t}$-type $(2,0)$. Recall that $\operatorname{dim}_{\mathbb{C}} X_{t}=3$ for all $t$. Suppose that $X_{t}$ were balanced for some $t=t_{12} \neq 0$ satisfying $\left|t_{12}\right|<\varepsilon$ with $\varepsilon>0$ small. Then there would exist a balanced metric $\omega_{t}>0$ on $X_{t}$. Thus $\Omega_{t}:=\omega_{t}^{2}$ would be a $C^{\infty}(2,2)$-form on $X_{t}$ satisfying

$$
\Omega_{t}>0, \quad d \Omega_{t}=0 .
$$

In this case we would have:

$$
0=\int_{X_{t}} d \Omega_{t} \wedge i \bar{t} \varphi_{3}(t)=-\int_{X_{t}} \Omega_{t} \wedge i \bar{t} d \varphi_{3}(t)=|t|^{2} \int_{X_{t}} \Omega_{t} \wedge i \varphi_{2}(t) \wedge \overline{\varphi_{2}(t)} .
$$


Indeed, the first identity above follows from $d \Omega_{t}=0(c f$. (3.52)), the second one follows from Stokes's theorem, while the third one follows from formula (3.51) for $d \varphi_{3}(t)$ and the fact that the $(2,0)$-component $-\varphi_{1}(t) \wedge \varphi_{2}(t)$ is annihilated when wedged with the $(2,2)$-form $\Omega_{t}$.

Now $\Omega_{t}>0$ and $i \varphi_{2}(t) \wedge \overline{\varphi_{2}(t)} \geq 0$, hence $\Omega_{t} \wedge i \varphi_{2}(t) \wedge \overline{\varphi_{2}(t)} \geq 0$ at every point of $X_{t}$. It follows that the right-hand term in (3.53) is non-negative. However, since it must vanish by the first identity in (3.53), the (3, 3)-form $\Omega_{t} \wedge i \varphi_{2}(t) \wedge \overline{\varphi_{2}(t)}$ must vanish identically on $X_{t}$, hence so must the $(1,1)$-form $i \varphi_{2}(t) \wedge \overline{\varphi_{2}(t)}$. This can only happen if $\varphi_{2}(t)$ vanishes identically on $X_{t}$, which is impossible since $\varphi_{2}(t)=$ $d \zeta_{2}(t)$ and $\zeta_{2}(t)$ is a holomorphic coordinate on $X_{t}$ if $\varepsilon$ is small enough. This provides the desired contradiction.

Therefore $X_{t}$ cannot be balanced for any $t=t_{12} \neq 0$ if $t_{i \lambda}=0$ for all $(i, \lambda) \neq$ $(1,2)$ and $\varepsilon>0$ is small. The proof is complete.

It is by means of this Proposition 3.13 that Alessandrini and Bassanelli proved Theorem 3.3: they observed that the fibres along one particular direction among the 6 directions available in the base space of the Kuranishi family of the Iwasawa manifold prove the non-openness of the balanced property under holomorphic deformations.

We now make the following:

Observation 3.14. (Implicit in [37]) In the Kuranishi family of the Iwasawa manifold, the Frölicher spectral sequence does not degenerate at $E_{1}$ (hence the $\partial \bar{\partial}$-lemma does not hold) on any fibre $X_{t}$ corresponding to parameters such that $t_{i \lambda}=0$ for all $(i, \lambda) \neq(1,2)$ and $t:=t_{12}$ satisfies $\left|t_{12}\right|<\varepsilon$ with $\varepsilon>0$ small enough.

Proof. We have seen in (3.5) that the second Betti number of the Iwasawa manifold is $b_{2}=8$. By the $C^{\infty}$-triviality of the family, all the fibres have the same Betti numbers. On the other hand, Nakamura concludes from his calculations reproduced above (via standard reasoning like that exemplified above between formulae (3.3) and (3.5)) that the Hodge numbers of weight 2 of any fibre $X_{t}$ corresponding to parameters such that $t_{i \lambda}=0$ for all $(i, \lambda) \neq(1,2)$ and $t:=t_{12} \neq 0$ are (see [37, table on page 96] for the case (ii) when $D(t)=0$ and $\left(t_{11}, t_{12}, t_{21}, t_{22}\right) \neq$ $(0,0,0,0)])$

$$
h^{2,0}(t)=h^{0,2}(t)=2, \quad h^{1,1}(t)=5
$$

Thus we see that for any such fibre $X_{t}$ with $t:=t_{12} \neq 0$, we have:

$$
b_{2}=8<h^{2,0}(t)+h^{1,1}(t)+h^{0,2}(t)=9 .
$$

Hence the conclusion follows. Notice that for fibres as above with $t:=t_{12} \neq 0$, Nakamura's table gives $b_{1}=4=2+2=h^{1,0}(t)+h^{0,1}(t)$, while we have seen in Observation 3.11 that for $X_{0}$ we have $b_{1}=4<3+2=h^{1,0}(0)+h^{0,1}(0)$. 


\subsection{The desired examples of $s G$ manifolds}

We can now conclude this section by exhibiting the desired examples of sG manifolds showing the difference between, on one hand, the sG property and, on the other hand, the (combined) balanced and $\partial \bar{\partial}$-lemma properties. It suffices to bring together Theorem 3.1, Theorem 3.3 and Observation 3.14:

Theorem 3.15. Let $\left(X_{t}\right)_{t}$ be the Kuranishi family of the Iwasawa manifold $X=X_{0}$, $t=\left(t_{i \lambda}\right)_{1 \leq i \leq 3,1 \leq \lambda \leq 2}$. Then, for parameters such that $t_{i \lambda}=0$ for all $(i, \lambda) \neq(1,2)$, $X_{t}$ is a strongly Gauduchon manifold that is not balanced and whose Frölicher spectral sequence does not degenerate at $E_{1}$ (hence the $\partial \bar{\partial}$-lemma does not hold) for any $t=t_{12} \neq 0$ satisfying $\left|t_{12}\right|<\varepsilon$ if $\varepsilon>0$ is small enough.

Proof. Since the Iwasawa manifold is balanced (cf. Corollary 3.6), it is also an sG manifold. Since the sG property is open under holomorphic deformations ( $c f$. Theorem 3.1), all sufficiently nearby fibres $X_{t}$ in the Kuranishi family of the Iwasawa manifold $X_{0}$ are again sG manifolds. However, by the observation of Alessandrini and Bassanelli ( $c f$. Proposition 3.13), the fibres $X_{t}$ corresponding to parameters for which $t_{i \lambda}=0$ for all $(i, \lambda) \neq(1,2)$ are not balanced if $t:=t_{12} \neq 0$ is sufficiently close to 0 . By Observation 3.14, the Frölicher spectral sequence does not degenerate at $E_{1}$, hence the $\partial \bar{\partial}$-lemma does not hold, on any of these fibres.

\section{The Eastwood-Singer construction}

In Subsection 4.2 of this section we give an outline of the Eastwood-Singer proof [16] of part (b) of Theorem 1.24 asserting the non-closedness of the Frölicher degeneration at $E_{1}$ under holomorphic deformations. All the fibres of the holomorphic family they construct in their example are twistor spaces (hence in particular compact complex 3 -folds). We also outline in 4.3 the proof given in [49, Theorem $1.25]$. We conclude by raising a few natural questions in 4.4 about other deformational stability properties of manifolds satisfying the $\partial \bar{\partial}$-lemma to which twistor spaces might hold the key.

\subsection{Background on twistor spaces}

We briefly recall the barest essentials of Penrose's twistor space theory [40] for which the standard mathematical reference is [1]. Given a compact oriented Riemannian manifold $(M, g)$ of real dimension 4 , for every point $x \in M$ one defines $L_{x}$ to be the set of all complex structures $J_{x}$ on the tangent space $T_{x} M$ such that $J_{x}$ is orthogonal with respect to $g_{x}$ and $J_{x}$ induces the negative (i.e. opposite to the given one) orientation on $T_{x} M$. Orthogonality with respect to $g_{x}$ for a complex structure $J_{x}: T_{x} M \rightarrow T_{x} M$ means, as usual, that

$$
g_{x}\left(J_{x} u, J_{x} v\right)=g_{x}(u, v), \quad \text { for all } u, v \in T_{x} M,
$$


i.e. $J_{x}$ is required to be a $g_{x}$-isometry of $T_{x} M$. When $x$ varies in $M$, the union $Z$ (which depends only on the conformal class $[g]$ of Riemannian metrics on $M$ but not on the actual representative $g$ ) of all twistor lines $L_{x}$ has a natural structure as a $C^{\infty}$ manifold of real dimension 6 and a natural almost complex structure. The almost complex structure is integrable if and only if the conformal structure $[g]$ of $M$ is self-dual. Recall that for an arbitrary-dimensional Riemannian manifold $(M, g)$, the curvature tensor $R$ decomposes as

$$
R=W+\rho,
$$

where $W$ is the Weyl tensor depending only on the conformal class $[g]$. Peculiar to the case when $M$ has real dimension 4 is a further decomposition of the Weyl tensor as

$$
W=W_{+}+W_{-},
$$

where $W_{+}$is the self-dual component and $W_{-}$is the anti-self-dual component. Reversing the orientation of $M$ permutes $W_{+}$and $W_{-}$. The conformal structure $[g]$ of $M$ is said to be self-dual if the associated Weyl tensor reduces to its self-dual component $W_{+}$(i.e. $W_{-}=0$ ).

One thus gets the Penrose correspondence between self-dual compact connected oriented $C^{\infty}$ real 4-manifolds $M$ and the associated twistor spaces $Z$ (which are compact complex 3-manifolds). The natural projection $v: Z \rightarrow M$ can be identified with the unit sphere subbundle of the rank-three real vector bundle of anti-self-dual 2-forms on $M$. Every twistor line $v^{-1}(x)=L_{x}$ is isomorphic to the complex projective line $\mathbb{P}^{1}$.

For every $r$, denote by $\Lambda^{r}:=C_{r}^{\infty}(M, \mathbb{C})$ the space of $C^{\infty}$ complex-valued $r$-forms on $M$. Recall that the Hodge star operator of the Riemannian metric $g$ of $M$ acting on 2-forms

$$
\star: \Lambda^{2} \rightarrow \Lambda^{2}
$$

satisfies $\star^{2}=1$. Hence it induces a direct-sum splitting

$$
\Lambda^{2}=\Lambda_{+}^{2} \oplus \Lambda_{-1}^{2}
$$

into its \pm 1 -eigenspaces. The 2-forms $u \in \Lambda_{+}^{2}$ (i.e. $\star u=u$ ) are termed self-dual, while the 2-forms $u \in \Lambda_{-}^{2}$ (i.e. $\star u=-u$ ) are termed anti-self-dual. If the Weyl curvature tensor $W$ is viewed as a bundle-valued 2-form, its components satisfy $\star W_{+}=W_{+}$and respectively $\star W_{-}=-W_{-}$. One gets a corresponding splitting of the differential operator $d$ acting on 1-forms of $M$ :

$$
\Lambda^{1 \stackrel{d=d_{+}+d_{-}}{\longrightarrow}} \Lambda^{2}=\Lambda_{+}^{2} \oplus \Lambda_{-}^{2}
$$

that induces cohomology groups $H_{+}^{2}(M, \mathbb{C})$ and $H_{-}^{2}(M, \mathbb{C})$. 


\subsection{The results of Eastwood and Singer}

For a fixed compact connected oriented $C^{\infty}$ manifold $M$ of real dimension 4 endowed with a self-dual Riemannian metric $g$, Eastwood and Singer establish the following general facts about the associated twistor space $Z$; the details may be found in [16, Section 2, 3, 4.]:

Fact 1. Given that the sheaves $\Omega^{1}, \Omega^{2}$ and $\Omega^{3}$ of germs of holomorphic 1,2 and respectively 3-forms on $Z$ are explicitly given, on every twistor line $L=L_{x}=\mathbb{P}^{1}$, by the formulae

$$
\begin{aligned}
& \Omega_{\mid L}^{1} \simeq \mathcal{O}(-2) \oplus \mathcal{O}(-1) \oplus \mathcal{O}(-1), \\
& \Omega_{\mid L}^{2} \simeq \mathcal{O}(-3) \oplus \mathcal{O}(-3) \oplus \mathcal{O}(-2), \\
& \Omega_{\mid L}^{3} \simeq \mathcal{O}(-4),
\end{aligned}
$$

we see that the restricted bundles $\Omega_{\mid L}^{1}, \Omega_{\mid L}^{2}, \Omega_{\mid L}^{3}$ have no non-trivial sections over $L$. Since $Z$ is fibred by projectives lines $L=L_{x}=\mathbb{P}^{1}$, it follows that the vector bundles $\Omega^{1}, \Omega^{2}, \Omega^{3}$ have no non-trivial global holomorphic sections over $Z$, hence

$$
H^{1,0}(Z, \mathbb{C})=0, \quad H^{2,0}(Z, \mathbb{C})=0, \quad H^{3,0}(Z, \mathbb{C})=0 .
$$

By Serre's duality, one also gets

$$
H^{2,3}(Z, \mathbb{C})=0, \quad H^{1,3}(Z, \mathbb{C})=0, \quad H^{0,3}(Z, \mathbb{C})=0 .
$$

Since $Z$ and $M$ are compact, one infers that

$$
H^{0,0}(Z, \mathbb{C})=H^{0}(M, \mathbb{C})=\mathbb{C} \text { and } H^{3,3}(Z, \mathbb{C})=H^{4}(M, \mathbb{C})=\mathbb{C},
$$

where the latter set of identities follows from the former by Serre duality on $Z$ and Poincaré duality on $M$.

Fact 2. The Penrose transform relating analytic cohomology on the twistor space $Z$ to solutions of differential equations on the base manifold $M$ is used to see the following:

- For all $q=0,1,2,3, H^{0, q}(Z, \mathbb{C})$ is canonically isomorphic to the $q^{\text {th }}$ cohomology of the complex

$$
\Lambda^{0} \longrightarrow \Lambda^{1} \stackrel{d_{-}}{\longrightarrow} \Lambda_{-}^{2} \longrightarrow 0
$$

- For all $q=0,1,2,3, H^{3, q}(Z, \mathbb{C})$ is canonically isomorphic to the $q^{\text {th }}$ cohomology of the complex

$$
0 \longrightarrow \Lambda_{-}^{2} \stackrel{d}{\longrightarrow} \Lambda^{3} \longrightarrow \Lambda^{4} .
$$

It follows as a corollary that

$$
\begin{array}{ll}
H^{0,1}(Z, \mathbb{C})=H^{1}(M, \mathbb{C}), & H^{0,2}(Z, \mathbb{C})=H_{-}^{2}(M, \mathbb{C}) \\
H^{3,1}(Z, \mathbb{C})=H_{-}^{2}(M, \mathbb{C}), & H^{3,2}(Z, \mathbb{C})=H^{3}(M, \mathbb{C}) .
\end{array}
$$


Fact 3. The Penrose transform of the vector bundles associated with $\Omega^{1}$ and $\Omega^{2}$ yields a commutative diagram with exact rows ( $c f$. [16, Proposition 3.3]) from which it follows that:

- $H^{1,1}(Z, \mathbb{C})$ can be identified with the set of pairs $(f, \rho) \in \Lambda^{0} \oplus \Lambda_{+}^{2}$ satisfying the equation

$$
\mathcal{D} d f-d \rho=0 \text { on } M,
$$

where the operator $\mathcal{D}: \Lambda^{1} \longrightarrow \Lambda^{3}$ is defined by

$$
\omega_{b} \mapsto\left(\nabla^{a} \nabla^{b}+2 R^{a b}-\frac{2}{3} R g^{a b}\right) \omega_{b},
$$

while $\nabla$ denotes the Levi-Civita connection, $R_{a b}$ denotes the Ricci curvature and $R$ denotes the scalar curvature of $(M, g)$;

- For $E_{2}^{1,1}$ featuring in the Frölicher spectral sequence of $Z$ at $E_{2}$ level, we always have the canonical isomorphism of $\mathbb{C}$-vector spaces:

$$
E_{2}^{1,1}:=\operatorname{ker}\left(d_{1}=\partial: H^{1,1}(Z, \mathbb{C}) \rightarrow H^{2,1}(Z, \mathbb{C})\right) \simeq H^{0}(M, \mathbb{C}) \oplus H_{+}^{2}(M, \mathbb{C}),
$$

hence $\operatorname{dim}_{\mathbb{C}} E_{2}^{1,1}=1+b_{+}(M)$, where $b_{+}(M):=\operatorname{dim}_{\mathbb{C}} H_{+}^{2}(M, \mathbb{C})$.

$\left(\right.$ Clearly $\operatorname{dim}_{\mathbb{C}} H^{0}(M, \mathbb{C})=1$ by compactness and connectedness of $M$.)

Fact 4. By [24], there exists a $(1,1)$-form $h$ on $Z$ such that $d h=0$ and $h_{\mid L}$ generates $H^{2}(L, \mathbb{C})$ for every twistor line $L=L_{x}=v^{-1}(x) \subset Z$. Using the Leray-Hirsch theorem, we get isomorphisms

$$
H^{r-2}(M, \mathbb{C}) \oplus H^{r}(M, \mathbb{C}) \simeq H^{r}(Z, \mathbb{C}), \quad 0 \leq r \leq 6,
$$

induced by $\Lambda^{r-2} \oplus \Lambda^{r} \ni(\alpha, \beta) \mapsto h \wedge v^{\star} \alpha+v^{\star} \beta \in C_{r}^{\infty}(Z, \mathbb{C})$.

The conclusion of these four facts is that for any twistor space $Z$ we always have $E_{2}(Z)=E_{\infty}(Z)$ (i.e. the Frölicher spectral sequence degenerates at the latest at $E_{2}$ level), while the degeneration at $E_{1}$ level depends exclusively on one arrow. Recall that for any complex manifold, the $E_{1}$ level of the Frölicher spectral sequence is given by the Dolbeault cohomology groups $\left(i . e . E_{1}^{p, q}=H^{p, q}\right.$ for all $p, q)$ and the $d_{1}$ arrows are induced by $\partial$ :

$$
E_{1}^{p, q} \stackrel{d_{1}=\partial}{\longrightarrow} E_{1}^{p+1, q} \text { for all } p, q
$$

When $Z$ is a twistor space, it follows from the above facts (1) - (4) that all arrows $d_{1}=\partial: E_{1}^{p, 0} \rightarrow E_{1}^{p+1,0}$ are zero when $q=0$ (hence also when $q=3$ by duality), 
while the part of the $E_{1}$ level of the Frölicher spectral sequence corresponding to $q=1$ reads:

$$
\begin{aligned}
& 0 \rightarrow H^{0,1}(Z, \mathbb{C}) \stackrel{d_{1}=0}{\longrightarrow} H^{1,1}(Z, \mathbb{C}) \stackrel{d_{1}}{\rightarrow} H^{2,1}(Z, \mathbb{C}) \stackrel{d_{1}=0}{\longrightarrow} H^{3,1}(Z, \mathbb{C}) \rightarrow 0 \\
& H^{1}(M, \mathbb{C}) \\
& H_{-}^{2}(M, \mathbb{C}) \text {. }
\end{aligned}
$$

Since the part corresponding to $q=2$ at $E_{1}$ level is dual to that for $q=1$, we see that $E_{1}(Z)=E_{\infty}(Z)$ if and only if the middle arrow above, i.e.

$$
d_{1}=\partial: H^{1,1}(Z, \mathbb{C}) \rightarrow H^{2,1}(Z, \mathbb{C}),
$$

vanishes. It is clear that the kernel of the arrow (4.8) equals $E_{2}^{1,1}(c f$. (4.7)) since the arrow preceding it in the last complex vanishes. We get the following:

Conclusion 4.1 (cf. [16, page 653-662]). For any twistor space $v: Z \rightarrow M$, $H^{0}(M, \mathbb{C}) \oplus H_{+}^{2}(M, \mathbb{C})$ injects canonically into $H^{1,1}(Z, \mathbb{C})$.

Moreover, the Frölicher spectral sequence of $Z$ degenerates at $E_{1}$ if and only if $H^{1,1}(Z, \mathbb{C})$ is isomorphic to $H^{0}(M, \mathbb{C}) \oplus H_{+}^{2}(M, \mathbb{C})$ iff $h^{1,1}(Z)=1+b_{+}(M)$

To construct the actual examples that prove part $(b)$ of Theorem 1.24, Eastwood and Singer [16, Section 5] go on to choose $M$ to be a compact complex surface endowed with a Kähler metric $\omega$ of zero scalar curvature. By [32], any such $M$ that has been given the conjugate orientation is self-dual, hence $M$ possesses a twistor space $Z$.

On the other hand, for any compact Kähler manifold $(X, \omega)$, the Lichnerowicz operator (cf.e.g. [7]) is defined on functions by

$$
\mathcal{L}: C^{\infty}(X, \mathbb{C}) \rightarrow C^{\infty}(X, \mathbb{C}), \quad \mathcal{L}(f):=\Delta^{2} f+\left\langle\left\langle d d^{c} f, \operatorname{Ric} \omega\right\rangle,,\right.
$$

where $\Delta$ is the Laplacian and Ric $\omega$ is the Ricci form of $\omega$. A result of Lichnerowicz (cf. e.g. [7, Proposition 2.151]]) guarantees that when the scalar curvature of $(X, \omega)$ is constant, there is an isomorphism

$$
u(X) / u_{0}(X) \simeq(\operatorname{ker} \mathcal{L}) / \mathbb{C},
$$

where $u(X)$ denotes the complex Lie algebra of holomorphic vector fields on $X$, while $u_{0}(X)$ denotes the Abelian Lie algebra of parallel such vector fields.

It turns out that when $(X, \omega)=(M, \omega)$ is a compact Kähler complex surface of zero scalar curvature, the operator $\mathcal{D}$ of (4.6) (defining equation (4.5) on $M$ which characterises the Dolbeault cohomology group $H^{1,1}(Z, \mathbb{C})$ ) relates to the Lichnerowicz operator as follows ( $c f$. [16, page 662]):

$$
d \mathcal{D} d=\mathcal{L},
$$

after identification of 4-forms with functions on $M$ via the volume form. 
This leads to the following consequence of the main theorem of [16]:

Theorem 4.2 ([16, Theorem 5.3.]). Let $(M, \omega)$ be a compact Kähler complex surface of zero scalar curvature. If $Z$ is the twistor space of $M$, then

$$
H^{1,1}(Z, \mathbb{C})=H^{0}(M, \mathbb{C}) \oplus H_{+}^{2}(M, \mathbb{C}) \oplus \frac{\{\text { holomorphic vector fields on } M\}}{\{\text { parallel vector fields on } M\}} .
$$

Since we always have $E_{2}^{1,1} \simeq H^{0}(M, \mathbb{C}) \oplus H_{+}^{2}(M, \mathbb{C})=\mathbb{C} \oplus H_{+}^{2}(M, \mathbb{C})$ (cf. (4.7)), we see by Conclusion 4.1 that the Frölicher spectral sequence of $Z$ degenerates at $E_{1}$ if and only if all holomorphic vector fields on $M$ are parallel.

The simplest case to which Eastwood and Singer apply their results is that of $M:=\Sigma_{g} \times \mathbb{P}^{1}$, where $\Sigma_{g}$ is any compact complex curve of genus $g \geq 2$ endowed with the Poincare metric $\omega_{P}$ (which is of constant curvature -1 ) and the complex projective line $\mathbb{P}^{1}$ is endowed with the metric $\omega_{\mathbb{P}^{1}}:=2 \omega_{F S}$ (which is of constant curvature +1 if $\omega_{F S}$ denotes the Fubini-Study metric). The metric $\omega$ induced on the product compact complex surface $M=\Sigma_{g} \times \mathbb{P}^{1}$ is a Kähler metric of zero scalar curvature.

Proposition 4.3 ([16, page 663]). If $M=\Sigma_{g} \times \mathbb{P}^{1}$ has been given the Kähler metric of zero scalar curvature $\omega=\omega_{P} \oplus \omega_{\mathbb{P} 1}$, the Frölicher spectral sequence of the twistor space $Z$ of $M$ does not degenerate at $E_{1}$.

Proof. We have seen in (4.5) that

$$
E_{1}^{1,1}=H^{1,1}(Z, \mathbb{C}) \simeq\left\{(f, \rho) \in \Lambda^{0} \oplus \Lambda_{+}^{2} ; \mathcal{D} d f=d \rho \text { on } M\right\} .
$$

On $M=\Sigma_{g} \times \mathbb{P}^{1}$, we have

$$
\mathcal{D} d f=\left(\nabla^{a} \nabla^{b}+2 R^{a b}\right) \nabla_{b} f
$$

because the scalar curvature $R \equiv 0$. If a $C^{\infty}$ function $f: \Sigma_{g} \times \mathbb{P}^{1} \rightarrow \mathbb{C}$ depends only on the $\mathbb{P}^{1}$ variable, we get:

$$
\begin{aligned}
\mathcal{D} d f & =\left(\nabla^{i} \nabla^{j}+2 \omega^{i j}\right) \nabla_{j} f=\nabla^{i}\left(\nabla^{j} \nabla_{j} f\right)+2 \omega^{i j} \nabla_{j} f \\
& =\nabla^{i}(-\Delta+2) f,
\end{aligned}
$$

where $\nabla_{j}$ is the Chern connection and $\Delta=-\nabla^{j} \nabla_{j}$ is the Laplacian on $\mathbb{P}^{1}$, while $\omega=\left(\omega_{i j}\right)_{i, j}$ locally on $\mathbb{P}^{1}$ and the identity $R^{i j}=\omega^{i j}$ holds because Ric $\omega=\omega$ on $\mathbb{P}^{1}$. Using now the well-known fact that 2 is an eigenvalue of the Laplacian on $\mathbb{P}^{1}$ with eigenspace $E_{\Delta}(2)$ of complex dimension 3 , we get $\left\{C^{\infty}\right.$ functions $\left.f: M \rightarrow \mathbb{C} ; \mathcal{D} d f=0\right\} \supset\{$ Constants $\} \oplus E_{\Delta}(2)=\mathbb{C} \oplus E_{\Delta}(2)$, hence $\operatorname{dim}_{\mathbb{C}}\left\{C^{\infty}\right.$ functions $\left.f: M \rightarrow \mathbb{C} ; \mathcal{D} d f=0\right\} \geq 4$. Thus (4.10) yields

$$
h^{1,1}(Z)=\operatorname{dim}_{\mathbb{C}} H^{1,1}(Z, \mathbb{C}) \geq 4+\operatorname{dim}_{\mathbb{C}}\left\{\rho \in \Lambda_{+}^{2} ; d \rho=0\right\}=4+b_{+}(M) .
$$

As $\operatorname{dim}_{\mathbb{C}} E_{2}^{1,1}=1+b_{+}(M)$ by (4.7), the claim follows from Conclusion 4.1. 
It is obvious that $M=\Sigma_{g} \times \mathbb{P}^{1}$ is the ruled surface $\mathbb{P}\left(E_{0}\right)$ associated with the trivial rank-two vector bundle $E_{0}:=\Sigma_{g} \times \mathbb{C}^{2} \rightarrow \Sigma_{g}$. This suggests a natural way of constructing a holomorphic family of compact complex surfaces $\left(M_{t}\right)_{t \in \Delta}$ such that $M_{0}=M=\Sigma_{g} \times \mathbb{P}^{1}$ for which we can hope to ensure that $E_{1}\left(Z_{t}\right)=E_{\infty}\left(Z_{t}\right)$ for all $t \in \Delta^{\star}$ in the associated family of twistor spaces $\left(Z_{t}\right)_{t \in \Delta}$ : take $M_{t}:=\mathbb{P}\left(E_{t}\right)$ when we have found a suitable family of rank-two holomorphic vector bundles $\left(E_{t}\right)_{t \in \Delta}$ over $\Sigma_{g}$ with the trivial bundle $E_{0}:=\Sigma_{g} \times \mathbb{C}^{2}$ corresponding to $t=0$. The authors make clear the meaning of suitable in the following form:

Proposition 4.4 ([16, page 663-664]). If $E \rightarrow \Sigma_{g}$ is a stable rank-two holomorphic vector bundle with trivial determinant over a compact complex curve of genus $g \geq 2$, then the corresponding ruled surface $\mathbb{P}(E)$ satisfies:

(i) $\mathbb{P}(E)$ admits a Kähler metric of zero scalar curvature;

(ii) $\mathbb{P}(E)$ has no non-zero holomorphic vector fields.

Proof. Eastwood and Singer deduce the above statements from classical results of Narasimhan-Seshadri [39] and Narasimhan-Ramanan [38].

By [39], any stable rank-two holomorphic vector bundle on $\Sigma_{g}$ (with $g \geq 2$ ) arises from a representation of $\pi_{1}\left(\Sigma_{g}\right)$ into $S U_{2}$, hence the corresponding ruled surface is a quotient $\mathbb{P}(E)=\left(\mathbb{P}^{1} \times H\right) / \pi_{1}$ where $H$ is the upper half-plane and $\pi_{1}$ acts by isometries of the natural metric on $\mathbb{P}^{1} \times H$. This natural metric, obtained as the Riemannian product of the metric of curvatute +1 on $\mathbb{P}^{1}$ with that of curvature -1 on $H$, is Kähler and of zero scalar curvature, hence (i) follows.

It follows from [39] that any vector bundle as in the statement satisfies $H^{0}\left(\Sigma_{g}, S^{2} E^{\star}\right)=0$. Indeed, for any rank-two vector bundle $E$ there is an isomorphism of bundles (see e.g. the proof in [25, Proposition 3.3.]:

$$
\operatorname{End}_{0} E \simeq S^{2} E^{\star} \otimes \Lambda^{2} E=S^{2} E^{\star} \otimes \operatorname{det} E
$$

induced by the map $T$ which associates with every $A \in$ End $E$ the quadratic map $E \ni v \mapsto A v \wedge v \in \Lambda^{2} E$. The kernel of $T$ consists of the scalar endomorphisms (identified with $\mathbb{C}$ ), while $\operatorname{End}_{0} E$ denotes the traceless endomorphisms. It obviously satisfies End $E=\operatorname{End}_{0} E \oplus \mathbb{C}$. On the other hand, if $E$ is stable then $E$ is simple ( $c f$. [39, Corollary to Proposition 4.3.]), i.e. $\operatorname{dim}_{\mathbb{C}} H^{0}\left(\Sigma_{g}\right.$, End $\left.E\right)=$ 1 , which means that the only endomorphisms of $E$ are the scalar ones. Hence $H^{0}\left(\Sigma_{g}, \operatorname{End}_{0} E\right)=0$ when $E$ is stable. Thus, if $E$ is stable and det $E$ is trivial, we see by (4.11) that $H^{0}\left(\Sigma_{g}, S^{2} E^{\star}\right)=0$.

Now, an easy argument explained in [16] shows that $H^{0}\left(\mathbb{P}(E), T^{1,0} \mathbb{P}(E)\right) \simeq$ $H^{0}\left(\Sigma_{g}, S^{2} E^{\star}\right)$ for any rank-two holomorphic vector bundle $E \rightarrow \Sigma_{g}$ when $g \geq 2$. This is because any holomorphic vector field $\xi \in H^{0}\left(\mathbb{P}(E), T^{1,0} \mathbb{P}(E)\right)$ must be vertical. Indeed, the normal bundle of any fibre of $\mathbb{P}(E) \rightarrow \Sigma_{g}$ being trivial, the component of $\xi$ normal to any such fibre is constant along the fibre, hence it projects to a holomorphic vector field on $\Sigma_{g}$. Now any holomorphic vector field on $\Sigma_{g}$ must vanish because, since $g \geq 2, \Sigma_{g}$ embeds into a $g$-dimensional complex 
torus $\mathbb{C}^{g} / \Lambda$ whose flat metric induces a metric of negative curvature on $T^{1,0} \Sigma_{g}$ viewed as a holomorphic line subbundle of the tangent bundle of $\mathbb{C}^{g} / \Lambda$. Thus any $\xi \in H^{0}\left(\mathbb{P}(E), T^{1,0} \mathbb{P}(E)\right)$ is indeed vertical, hence the restriction of $\xi$ to any fibre of $\mathbb{P}(E) \rightarrow \Sigma_{g}$ defines a holomorphic vector field on the fibre $\mathbb{P}\left(E_{t}\right) \simeq \mathbb{P}^{1}$. Meanwhile, the holomorphic vector fields of $\mathbb{P}^{1}$ are the holomorphic sections of $-K_{\mathbb{P}^{1}}=\mathcal{O}_{\mathbb{P}^{1}}(2)$. Thus (ii) follows.

We now get an immediate corollary of Theorem 4.2 and Proposition 4.4. In view of Theorem 4.2, a weaker version of conclusion (ii) of Proposition 4.4 (with non-parallel in place of non-zero) would have sufficed.

Corollary 4.5 ([16, page 664]). Let $E \rightarrow \Sigma_{g}$ be a stable rank-two holomorphic vector bundle with trivial determinant over a compact complex curve of genus $g \geq 2$. Let $Z$ be the twistor space associated with the ruled surface $\mathbb{P}(E)$. Then the Frölicher spectral sequence of $Z$ degenerates at $E_{1}$.

Putting together Proposition 4.3 and Corollary 4.5, we see that it suffices to show that the trivial rank-two vector bundle $E_{0}=\Sigma_{g} \times \mathbb{C}^{2} \rightarrow \Sigma_{g}$ deforms to stable rank-two holomorphic vector bundles $E_{t} \rightarrow \Sigma_{g}$ with trivial determinant, $t \in \Delta^{\star}$. As explained before the statement of Proposition 4.4, associated with the family of bundles $\left(E_{t}\right)_{t \in \Delta}$ will be the family of ruled surfaces $\left(M_{t}:=\mathbb{P}\left(E_{t}\right)\right)_{t \in \Delta}$ whose corresponding family of twistor spaces $\left(Z_{t}\right)_{t \in \Delta}$ will provide the example proving the Eastwood-Singer part $(b)$ of Theorem 1.24: $E_{1}\left(Z_{t}\right)=E_{\infty}\left(Z_{t}\right)$ for all $t \neq 0$ by Corollary 4.5 , but $E_{1}\left(Z_{0}\right) \neq E_{\infty}\left(Z_{0}\right)$ by Proposition 4.3.

Theorem 4.6 ([16, page 664-665]) Given any compact complex curve $\Sigma_{g}$ of genus $g \geq 2$, the trivial rank-two vector bundle $E_{0}=\Sigma_{g} \times \mathbb{C}^{2} \rightarrow \Sigma_{g}$ deforms to stable rank-two holomorphic vector bundles with trivial determinant on $\Sigma_{g}$.

Proof. We give an outline of the proof found in [16] which, as mentioned there, is a modification of an argument from [38]. Fix an arbitrary point $x \in \Sigma_{g}$ and denote by $L_{x}$ the holomorphic line bundle on $\Sigma_{g}$ defined by $x$ (viewed as a divisor on the curve $\Sigma_{g}$ ). If $L_{x}^{-1}$ denotes the line bundle dual to $L_{x}$, consider the rank-two holomorphic vector bundles $E \rightarrow \Sigma_{g}$ that are non-trivial extensions of $L_{x}$ by $L_{x}^{-1}$. Equivalently, one considers short exact sequences of vector bundles on $\Sigma_{g}$ :

$$
0 \rightarrow L_{x}^{-1} \rightarrow E \rightarrow L_{x} \rightarrow 0
$$

that do not split holomorphically. There is a one-to-one correspondence between the equivalence classes of such non-trivial extensions and the non-zero classes $\left\{\beta^{\star}\right\} \in H^{0,1}\left(\Sigma_{g}, \operatorname{Hom}\left(L_{x}, L_{x}^{-1}\right)\right) \simeq H^{1}\left(\Sigma_{g}, \mathcal{O}\left(L_{x}^{-2}\right)\right)$, where $\beta^{\star}$ denotes the second fundamental form of extension (4.12). Any such $E$ has trivial determinant (since det $E=L_{x}^{-1} \otimes L_{x}$ ) and degree zero since $L_{x}$ has degree $+1, L_{x}^{-1}$ has degree -1 and the degree is additive in exact sequences. By [38, Lemma 5.1], any such $E$ can have no holomorphic line subbundles of positive degree, hence any such $E$ is at least semi-stable. To find stable vector bundles $E$ arising as extensions (4.12), 
it remains to rule out the existence of holomorphic line subbundles of degree zero in $E$.

Suppose that a rank-two holomorphic vector bundle $E$ given by an extension (4.12) contains a degree-zero holomorphic line subbundle $L \subset E$. By the proof of [38, Lemma 5.2], any such $L$ must be of the form $L=L_{x} \otimes L_{y}^{-1}$ for some point $y \in$ $\Sigma_{g}$ and [38, Lemma 5.2] asserts that the class $\left\{\beta^{\star}\right\}$ of extension (4.12) maps to the zero class in the cohomology group $H^{0,1}\left(\Sigma_{g}, \operatorname{Hom}\left(L, L_{x}^{-1}\right)\right)=H^{1}\left(\Sigma_{g}, \mathcal{O}\left(L_{x}^{-2} \otimes\right.\right.$ $\left.L_{y}\right)$ ) under the map

$$
H^{1}\left(\Sigma_{g}, \mathcal{O}\left(L_{x}^{-2}\right)\right) \rightarrow H^{1}\left(\Sigma_{g}, \mathcal{O}\left(L_{x}^{-2} \otimes L_{y}\right)\right) .
$$

Since (4.13) is a surjective linear mapping of complex vector spaces of dimensions $g+1$ and respectively $g$ thanks to Riemann-Roch, the kernel of (4.13) is a complex line $l_{y}$ in the $(g+1)$-dimensional vector space $H^{1}\left(\Sigma_{g}, \mathcal{O}\left(L_{x}^{-2}\right)\right)$. We conclude that $L=L_{x} \otimes L_{y}^{-1}$ is a holomorphic subbundle of $E$ iff

$$
\left\{\beta^{\star}\right\} \in \operatorname{ker}\left(H^{1}\left(\Sigma_{g}, \mathcal{O}\left(L_{x}^{-2}\right)\right) \rightarrow H^{1}\left(\Sigma_{g}, \mathcal{O}\left(L_{x}^{-2} \otimes L_{y}\right)\right)\right) \backslash\{0\}=l_{y} \backslash\{0\} .
$$

By [38, Lemma 3.3], proportional extension classes $\left\{\beta^{\star}\right\}$ and $\lambda\left\{\beta^{\star}\right\}$, with $\lambda \in \mathbb{C}^{\star}$, give rise to isomorphic bundles $E$ and $E_{\lambda}$. Now the punctured line $l_{y} \backslash\{0\}$ defines a point in the complex projective space $\mathbb{P} H^{1}\left(\Sigma_{g}, \mathcal{O}\left(L_{x}^{-2}\right)\right) \simeq \mathbb{P}^{g}$ and, when $y$ varies in $\Sigma_{g}$, we get an analytic mapping

$$
\Sigma_{g} \ni y \mapsto l_{y} \backslash\{0\} \in \mathbb{P} H^{1}\left(\Sigma_{g}, \mathcal{O}\left(L_{x}^{-2}\right)\right) \simeq \mathbb{P}^{g} .
$$

The conclusion is that an extension class $\left\{\beta^{\star}\right\} \in H^{1}\left(\Sigma_{g}, \mathcal{O}\left(L_{x}^{-2}\right)\right) \backslash\{0\}$ defines a holomorphic rank-two vector bundle $E \rightarrow \Sigma_{g}$ with trivial determinant that contains no degree-zero holomorphic line subbundles $L \subset E$ (hence $E$ is stable) if and only if the image of $\left\{\beta^{\star}\right\}$ in the projective space $\mathbb{P} H^{1}\left(\Sigma_{g}, \mathcal{O}\left(L_{x}^{-2}\right)\right) \simeq \mathbb{P}^{g}$ under the natural projection $H^{1}\left(\Sigma_{g}, \mathcal{O}\left(L_{x}^{-2}\right)\right) \backslash\{0\} \rightarrow \mathbb{P} H^{1}\left(\Sigma_{g}, \mathcal{O}\left(L_{x}^{-2}\right)\right)$ lies in the complement of the curve which is the image of the map (4.14). Since $g \geq 2$, the complement of a complex curve in the $g$-dimensional complex projective space $\mathbb{P}^{g}$ provides plenty of room for choice of deformations $E=E_{t}, t \neq 0$, of the trivial rank-two vector bundle $E_{0}$. Of course, $E_{0}$ corresponds to the trivial extension (4.12) or, equivalently, to the zero class $\left\{\beta^{\star}\right\} \in H^{1}\left(\Sigma_{g}, \mathcal{O}\left(L_{x}^{-2}\right)\right)$.

As explained before the statement of Theorem 4.6, this result provides the final argument to the proof of the Eastwood-Singer part (b) of Theorem 1.24. We have chosen to reproduce the approach of [16] in some detail because it throws up new stimulating questions of which we now mention just a few.

\subsection{Wu's deformation openness of the $\partial \bar{\partial}$-lemma property}

In this subsection we outline the main ideas in the proof given by C.-C. Wu in [49] to the fact that the $\partial \bar{\partial}$-lemma for compact complex manifolds is a deformation-open property ( $c f$. Theorem 1.25). 
The proof in [49] makes crucial use of a characterisation of the $\partial \bar{\partial}$-lemma that was given in [14] (cf. Proposition 4.8 below). It is cast in terms of the relations between the Bott-Chern and the Aeppli cohomology groups of a compact complex manifold $X\left(\operatorname{dim}_{\mathbb{C}} X=n\right)$ whose definitions (for all $p, q=0, \ldots, n$ ) we now recall:

$$
H_{B C}^{p, q}(X, \mathbb{C}):=\frac{\operatorname{ker} \partial \cap \operatorname{ker} \bar{\partial}}{\operatorname{Im}(\partial \bar{\partial})} \text { and } H_{A}^{p, q}(X, \mathbb{C}):=\frac{\operatorname{ker}(\partial \bar{\partial})}{\operatorname{Im} \partial+\operatorname{Im} \bar{\partial}} .
$$

(The operators $\partial, \bar{\partial}$ and $\partial \bar{\partial}$ featuring in the numerators act on $(p, q)$-forms, while those in the denominators arrive in the subspace of $(p, q)$-forms.)

In a similar way, one also standardly defines

$$
H_{\partial}^{p, q}(X, \mathbb{C}):=\frac{\operatorname{ker} \partial}{\operatorname{Im} \partial} \text { and } H_{\bar{\partial}}^{p, q}(X, \mathbb{C}):=\frac{\operatorname{ker} \bar{\partial}}{\operatorname{Im} \bar{\partial}} .
$$

(Of course, $H_{\bar{\partial}}^{p, q}(X, \mathbb{C})$ is the Dolbeault cohomology group of type $(p, q)$ that was earlier denoted by $H^{p, q}(X, \mathbb{C})$.)

For all $p, q=0, \ldots, n$, there is always a natural (canonical) linear map

$$
\begin{gathered}
H_{B C}^{p, q}(X, \mathbb{C}) \longrightarrow H_{A}^{p, q}(X, \mathbb{C}) \\
{[u]_{B C} \longmapsto[u]_{A}}
\end{gathered}
$$

mapping the Bott-Chern class of any $d$-closed $(p, q)$-form $u$ to the Aeppli class of $u$ (which makes sense since $\partial \bar{\partial} u=0$ whenever $\partial u=0$ and $\bar{\partial} u=0$ ). This map is always well defined but it need not be either injective or surjective on an arbitrary $X$. However, it is an isomorphism when $X$ satisfies the $\partial \bar{\partial}$-lemma. The converse also holds as Proposition 4.8 will implicitly show.

Before stating the $\partial \bar{\partial}$-lemma characterisation of [14], we introduce some ad hoc terminology:

Definition 4.7. For a given $k=0,1, \ldots, 2 n$, a given compact complex manifold $X$ (with $\operatorname{dim}_{\mathbb{C}} X=n$ ) is said to satisfy property:

$\left(A_{k}\right)$ if the natural map $H_{B C}^{p, q}(X, \mathbb{C}) \longrightarrow H_{A}^{p, q}(X, \mathbb{C})$ is injective for all $p, q$ such that $p+q=k$.

This property is clearly equivalent to property

$\left(A_{k}^{\prime}\right) \operatorname{ker} \partial \cap \operatorname{ker} \bar{\partial} \cap(\operatorname{Im} \partial+\operatorname{Im} \bar{\partial})=\operatorname{Im}(\partial \bar{\partial}), \quad$ for all $p, q$ s.t. $p+q=k$.

$\left(B_{k}\right)$ if the natural map $H_{B C}^{p, q}(X, \mathbb{C}) \longrightarrow H_{A}^{p, q}(X, \mathbb{C})$ is surjective for all $p, q$ such that $p+q=k$.

This property is clearly equivalent to property

$\left(B_{k}^{\prime}\right) \operatorname{Im} \partial+\operatorname{Im} \bar{\partial}+(\operatorname{ker} \partial \cap \operatorname{ker} \bar{\partial})=\operatorname{ker}(\partial \bar{\partial}), \quad$ for all $p, q$ s.t. $p+q=k$.

$\left(C_{k}\right)$ if the natural maps $H_{B C}^{p, q}(X, \mathbb{C}) \longrightarrow H_{\partial}^{p, q}(X, \mathbb{C})$ and $H_{B C}^{p, q}(X, \mathbb{C}) \longrightarrow H_{\bar{\partial}}^{p, q}(X, \mathbb{C})$ are injective for all $p, q$ such that $p+q=k$.

This property is clearly equivalent to the simultaneous occurence of 
$\left(C_{k}^{\prime}\right)$ (i) $\operatorname{Im} \partial \cap \operatorname{ker} \bar{\partial}=\operatorname{Im}(\partial \bar{\partial}) \quad$ and $\quad\left(C_{k}^{\prime}\right)($ ii) $\operatorname{Im} \bar{\partial} \cap \operatorname{ker} \partial=\operatorname{Im}(\partial \bar{\partial})$, for all $p, q$ such that $p+q=k$.

$\left(D_{k}^{\prime}\right)$ if (i) $\operatorname{Im} \bar{\partial}+\operatorname{ker} \partial=\operatorname{ker}(\partial \bar{\partial})$ and (ii) $\operatorname{Im} \partial+\operatorname{ker} \bar{\partial}=\operatorname{ker}(\partial \bar{\partial})$ for all $p, q$ such that $p+q=k$.

$\left(L_{k}\right)$ if the $\partial \bar{\partial}$-lemma holds on every space of forms $C_{p, q}^{\infty}(X, \mathbb{C})$ with $p+q=k$.

It is obvious that $\left(L_{k}\right)$ implies each of the other properties listed above and that $\left(L_{k}\right)$ is implied by the simultaneous occurence of these other properties. As already pointed out, the following equivalences are obvious

$$
\left(A_{k}\right) \Longleftrightarrow\left(A_{k}^{\prime}\right), \quad\left(B_{k}\right) \Longleftrightarrow\left(B_{k}^{\prime}\right), \quad\left(C_{k}\right) \Longleftrightarrow\left(C_{k}^{\prime}\right) .
$$

The inclusions $\supset$ in $\left(A_{k}^{\prime}\right), \subset$ in $\left(B_{k}^{\prime}\right), \supset$ in $\left(C_{k}^{\prime}\right)(\mathrm{i})$, (ii) and $\subset$ in $\left(D_{k}^{\prime}\right)$ (i), (ii) are obvious. The following statement is implicit in [14] and provides a key ingredient for the proof of Theorem 1.25 obtained in [49].

Proposition 4.8 (Contained in [14, Lemma 5.15]). Let $X$ be a compact complex manifold, $\operatorname{dim}_{\mathbb{C}} X=n$. For every $k=1, \ldots, 2 n$, the following equivalences hold:

$$
\left(L_{k}\right) \Longleftrightarrow\left(A_{k}\right) \Longleftrightarrow\left(C_{k}\right) \Longleftrightarrow\left(D_{k-1}^{\prime}\right) \Longleftrightarrow\left(B_{k-1}\right) \text {. }
$$

Proof. Fix an arbitrary $k \in\{1, \ldots, 2 n\}$. In view of what we have already noticed, it suffices to prove the equivalences

$$
\left(A_{k}^{\prime}\right) \Longleftrightarrow\left(C_{k}^{\prime}\right) \Longleftrightarrow\left(D_{k-1}^{\prime}\right) \Longleftrightarrow\left(B_{k-1}^{\prime}\right) .
$$

Proof of $\left(A_{k}^{\prime}\right) \Longrightarrow\left(C_{k}^{\prime}\right)$. Let $u \in C_{p, q}^{\infty}(X, \mathbb{C})$ (where $p+q=k$ ) such that $\bar{\partial} u=0$ and $u=\partial v$ for some $(p-1, q)$-form $v$. Then $u=\partial v+0 \in \operatorname{ker} \partial \cap \operatorname{ker} \bar{\partial} \cap(\operatorname{Im} \partial+$ $\operatorname{Im} \bar{\partial})$. Then $\left(A_{k}^{\prime}\right)$ forces $u \in \operatorname{Im}(\partial \bar{\partial})$. This proves (i) of $\left(C_{k}^{\prime}\right)$. The proof of (ii) of $\left(C_{k}^{\prime}\right)$ is similar with $\partial$ and $\bar{\partial}$ reversed.

Proof of $\left(C_{k}^{\prime}\right) \Longrightarrow\left(A_{k}^{\prime}\right)$. Let $u \in C_{p, q}^{\infty}(X, \mathbb{C})$ (where $p+q=k$ ) such that $\partial u=0$, $\bar{\partial} u=0$ and $u=\partial v+\bar{\partial} w$ for some $(p-1, q)$-form $v$ and some $(p, q-1)$-form $w$. Then we have:

- $\operatorname{Im} \partial \ni \partial v=u-\bar{\partial} w \in \operatorname{ker} \bar{\partial}$, hence $\partial v \in \operatorname{Im} \partial \cap \operatorname{ker} \bar{\partial}=\operatorname{Im}(\partial \bar{\partial})$, the last identity of subspaces being given by the hypothesis $\left(C_{k}^{\prime}\right)(i)$. Thus $\partial v \in \operatorname{Im}(\partial \bar{\partial})$.

- $\operatorname{Im} \bar{\partial} \ni \bar{\partial} w=u-\partial v \in \operatorname{ker} \partial$, hence $\bar{\partial} w \in \operatorname{Im} \bar{\partial} \cap \operatorname{ker} \partial=\operatorname{Im}(\partial \bar{\partial})$, the last identity of subspaces being given by the hypothesis $\left(C_{k}^{\prime}\right)(i i)$. Thus $\bar{\partial} w \in \operatorname{Im}(\partial \bar{\partial})$.

It is now clear that $u=\partial v+\bar{\partial} w \in \operatorname{Im}(\partial \bar{\partial})$. This proves $\left(A_{k}^{\prime}\right)$.

Proof of $\left(C_{k}^{\prime}\right) \Longrightarrow\left(D_{k-1}^{\prime}\right)$. Let $u \in C_{r, s}^{\infty}(X, \mathbb{C})$ (where $r+s=k-1$ ) such that $\partial \bar{\partial} u=0$. Then: 
- $\partial u$ is a $k$-form of type $(r+1, s)$ and $\partial u \in \operatorname{ker} \bar{\partial} \cap \operatorname{Im} \partial=\operatorname{Im}(\partial \bar{\partial})$, the last identity of subspaces being given by the hypothesis $\left(C_{k}^{\prime}\right)(\mathrm{i})$. Hence $\partial u=\partial \bar{\partial} \zeta$ for some $(r, s-1)$-form $\zeta$. This amounts to $\partial(u-\bar{\partial} \zeta)=0$ or again to $u-\bar{\partial} \zeta \in \operatorname{ker} \partial$.

We get $u=\bar{\partial} \zeta+(u-\bar{\partial} \zeta) \in \operatorname{Im} \bar{\partial}+\operatorname{ker} \partial$. This proves $\left(D_{k-1}^{\prime}\right)(\mathrm{i})$.

- $\bar{\partial} u$ is a $k$-form of type $(r, s+1)$ and $\bar{\partial} u \in \operatorname{ker} \partial \cap \operatorname{Im} \bar{\partial}=\operatorname{Im}(\partial \bar{\partial})$, the last identity of subspaces being given by the hypothesis $\left(C_{k}^{\prime}\right)$ (ii). Hence $\bar{\partial} u=\partial \bar{\partial} w$ for some $(r-1, s)$-form $w$. This amounts to $\bar{\partial}(u+\partial w)=0$ or again to $u+\partial w \in \operatorname{ker} \bar{\partial}$.

We get $u=-\partial w+(u+\partial w) \in \operatorname{Im} \partial+\operatorname{ker} \bar{\partial}$. This proves $\left(D_{k-1}^{\prime}\right)($ ii) .

Proof of $\left(D_{k-1}^{\prime}\right) \Longrightarrow\left(C_{k}^{\prime}\right)$. Let $u \in C_{p, q}^{\infty}(X, \mathbb{C})$ (where $p+q=k$ ) such that $\bar{\partial} u=0$ and $u=\partial v$ for some $(k-1)$-form $v$ of type $(p-1, q)$. Then $0=\bar{\partial} u=-\partial \bar{\partial} v$, hence $v \in \operatorname{ker}(\partial \bar{\partial})$. Since $\operatorname{ker}(\partial \bar{\partial})=\operatorname{Im} \bar{\partial}+\operatorname{ker} \partial$ for $(k-1)$-forms by $\left(D_{k-1}^{\prime}\right)(\mathrm{i})$, we can find a $(p-1, q-1)$-form $w$ and a $(p-1, q)$-form $\zeta$ such that

$$
v=\bar{\partial} w+\zeta \quad \text { and } \quad \partial \zeta=0
$$

Applying $\partial$, we get: $u=\partial v=\partial \bar{\partial} w$. Thus $u \in \operatorname{Im}(\partial \bar{\partial})$. This proves $\left(C_{k}^{\prime}\right)(\mathrm{i})$.

Reversing the roles of $\partial$ and $\bar{\partial}$, we get $\left(C_{k}^{\prime}\right)$ (ii) in a similar way from $\left(D_{k-1}^{\prime}\right)($ ii) .

Proof of $\left(D_{k-1}^{\prime}\right) \Longrightarrow\left(B_{k-1}^{\prime}\right)$. Let $u \in C_{r, s}^{\infty}(X, \mathbb{C})$ (where $r+s=k-1$ ) such that $\partial \bar{\partial} u=0$. Thanks to $\left(D_{k-1}^{\prime}\right)(i i)$, we can find an $(r-1, s)$-form $v$ and an $(r, s)$-form $w$ such that

$$
u=\partial v+w \text { and } \quad w \in \operatorname{ker} \bar{\partial} .
$$

Now since $\bar{\partial} w=0$, we also have $\partial \bar{\partial} w=0$. Hence by $\left(D_{k-1}^{\prime}\right)(i)$ we can write

$$
w=\bar{\partial} \zeta+\rho \quad \text { with } \quad \rho \in \operatorname{ker} \partial
$$

for some $(r, s-1)$-form $\zeta$ and some $(r, s)$-form $\rho$. We get: $\rho=w-\bar{\partial} \zeta$ and since $w, \bar{\partial} \zeta \in \operatorname{ker} \bar{\partial}$, we finally get $\rho \in \operatorname{ker} \bar{\partial}$. Given the choice of $\rho$, this implies that $\rho \in \operatorname{ker} \partial \cap \operatorname{ker} \bar{\partial}$.

Putting the bits together, we have

$$
u=\partial v+\bar{\partial} \zeta+\rho \in \operatorname{Im} \partial+\operatorname{Im} \bar{\partial}+(\operatorname{ker} \partial \cap \operatorname{ker} \bar{\partial}) .
$$

This proves $\left(B_{k-1}^{\prime}\right)$.

Proof of $\left(B_{k-1}^{\prime}\right) \Longrightarrow\left(D_{k-1}^{\prime}\right)$. This implication is trivial because $\operatorname{Im} \partial+(\operatorname{ker} \partial \cap$ $\operatorname{ker} \bar{\partial}) \subset \operatorname{ker} \partial$ and $\operatorname{Im} \bar{\partial}+(\operatorname{ker} \partial \cap \operatorname{ker} \bar{\partial}) \subset \operatorname{ker} \bar{\partial}$.

The Proposition is proved. 
Outline of proof of Wu's Theorem 1.25 (according to [49]). The main idea is to exploit the one-unit discrepancy between $k$ and $(k-1)$ in the Deligne-GriffithsMorgan-Sullivan characterisations given to the $\partial \bar{\partial}$-lemma in Proposition 4.8, namely the equivalences

$$
\left(L_{k}\right) \Longleftrightarrow\left(A_{k}\right) \Longleftrightarrow\left(B_{k-1}\right)
$$

This enables an argument by induction on the degree $k$ of forms. Note that the $\partial \bar{\partial}$ lemma always holds in a trivial way for $k=0$ (i.e. on functions). Fix an arbitrary holomorphic family of compact complex $n$-dimensional manifolds $\left(X_{t}\right)_{t \in \Delta}$. The proof falls into two steps.

Step 1. Assuming the $\partial \bar{\partial}$-lemma on $X_{0}$, Wu shows that the dimensions of the BottChern and Aeppli cohomology spaces remain constant in a small neighbourhood of $0 \in \Delta$, i.e.

$$
\begin{array}{ll}
\operatorname{dim}_{\mathbb{C}} H_{B C}^{p, q}\left(X_{t}, \mathbb{C}\right)=\operatorname{dim}_{\mathbb{C}} H_{B C}^{p, q}\left(X_{0}, \mathbb{C}\right) & \text { for all } t \in \Delta \text { sufficiently close to } 0 \\
\operatorname{dim}_{\mathbb{C}} H_{A}^{p, q}\left(X_{t}, \mathbb{C}\right)=\operatorname{dim}_{\mathbb{C}} H_{A}^{p, q}\left(X_{0}, \mathbb{C}\right) & \text { for all } t \in \Delta \text { sufficiently close to } 0 .
\end{array}
$$

This parallels the analogous results for the Dolbeault cohomology that are classical and known to hold under the weaker assumption that the Frölicher spectral sequence of $X_{0}$ degenerates at $E_{1}$.

Step 2. Suppose the $\partial \bar{\partial}$-lemma holds on $X_{0}$. Wu shows that the same thing holds on every sufficiently close fibre $X_{t}$ by induction on $k=0,1, \ldots, 2 n$. Suppose the $\partial \bar{\partial}$-lemma holds for the $k$-forms of $X_{t}$. We have to show that it then also holds for the $(k+1)$-forms of $X_{t}$.

The induction hypothesis is that $\left(L_{k}\right)$ holds on $X_{t}$ which, by Proposition 4.8 , is equivalent to $\left(A_{k}\right)$ holding on $X_{t}$. This means that the natural map $H_{B C}^{p, q}\left(X_{t}, \mathbb{C}\right) \longrightarrow$ $H_{A}^{p, q}\left(X_{t}, \mathbb{C}\right)$ is injective for all $p, q$ such that $p+q=k$.

On the other hand, $\operatorname{dim}_{\mathbb{C}} H_{B C}^{p, q}\left(X_{0}, \mathbb{C}\right)=\operatorname{dim}_{\mathbb{C}} H_{A}^{p, q}\left(X_{0}, \mathbb{C}\right)$ for all $p, q$ thanks to the $\partial \bar{\partial}$-lemma assumption on $X_{0}$ (see explanation above Definition 4.7 ). Since, by Step 1 , the dimensions of these spaces do not change when $t$ remains close to 0 , we still have

$$
\operatorname{dim}_{\mathbb{C}} H_{B C}^{p, q}\left(X_{t}, \mathbb{C}\right)=\operatorname{dim}_{\mathbb{C}} H_{A}^{p, q}\left(X_{t}, \mathbb{C}\right), \quad \text { for all } p, q \text { and all } t \text { near } 0 .
$$

Thus, for all $t$ near $0, H_{B C}^{p, q}\left(X_{t}, \mathbb{C}\right)$ and $H_{A}^{p, q}\left(X_{t}, \mathbb{C}\right)$ are finite-dimensional vector spaces of equal dimensions and the natural linear map between them is injective whenever $p+q=k$ by the induction hypothesis $\left(A_{k}\right)$. Then it must also be surjective, which means that property $\left(B_{k}\right)$ holds on $X_{t}$ for all $t$ close to 0 . By Proposition 4.8 , this is equivalent to property $\left(L_{k+1}\right)$, i.e. to the $\partial \bar{\partial}$-lemma for the $(k+1)$-forms of $X_{t}$. 


\subsection{Twistor spaces and the $\partial \bar{\partial}$-lemma}

The limiting behaviour under holomorphic deformations of compact complex manifolds satisfying the $\partial \bar{\partial}$-lemma seems to be shrouded in mystery. A likely guess is that this property may not be deformation-closed. Our expectation is that deformations of twistor spaces may hold the key. We take this opportunity to raise a few natural questions.

We start by recalling the following result of Gauduchon.

Theorem 4.9 ([20, Proposition 11.b, page 618]). Every twistor space is balanced $^{10}$. Moreover, this is the case for any self-dual metric on the corresponding four-manifold (i.e. a balanced metric is obtained on the twistor space from any self-dual metric in a given conformal class of the base four-manifold).

Combined with [ES93], this readily (re-)proves part $(a)$ of Theorem 1.11. Indeed, by Gauduchon's Theorem 4.9, the central fibre $Z_{0}$ in the Eastwood-Singer family is an sG manifold since, as a twistor space, it even has the stronger balanced property. However, $E_{1}\left(Z_{0}\right) \neq E_{\infty}\left(Z_{0}\right)$ as has been seen in Proposition 4.3. Recall that the Iwasawa manifold had provided another example proving $(a)$ of Theorem 1.11 ( $c f$. comments after Observation 3.11).

On the other hand, Hitchin's main result in [24] states that there exist only two Kähler twistor spaces: $\mathbb{P}^{3}$ and the space of flags in $\mathbb{C}^{3}$. Together with Gauduchon's Theorem 4.9 and Campana's result in [11] stating that a twistor space is Moishezon if and only if it is of class- $\mathcal{C}$, this fact will point to a relative lack of variation in the properties of twistor spaces.

However, combining Wu's Theorem 1.25 with the examples of Campana [10] and Lebrun-Poon [34] that proved the deformation non-openness of the class- $\mathcal{C}$ property ( $c f$. Theorem 1.18), we notice the first piece of evidence against the above mock "principle".

Observation 4.10. There exist twistor spaces satisfying the $\partial \bar{\partial}$-lemma that are not of class $\mathcal{C}$.

Indeed, in [10] and [34] families of twistor spaces $\left(Z_{t}\right)_{t \in \Delta}$ are constructed in which the central fibre $Z_{0}$ is a Moishezon $(=$ class $-\mathcal{C}$ ) twistor space (hence its algebraic dimension is maximal: $a\left(Z_{0}\right)=\operatorname{dim}_{\mathbb{C}} Z_{0}=3$ ), while all the nearby fibres can be chosen to be decidedly non-Moishezon $(=$ non-class- $\mathcal{C})$ twistor spaces, i.e. to have minimal algebraic dimension $a\left(Z_{t}\right)=0$ for $t \neq 0$. Now, the Moishezon central fibre $Z_{0}$ must satisfy the $\partial \bar{\partial}$-lemma, hence by Wu's Theorem 1.25 all the nearby fibres $Z_{t}$ also satisfy the $\partial \bar{\partial}$-lemma if $t \in \Delta^{\star}$ is sufficiently close to 0 . Thus any of these nearby fibres is an example of a twistor space that is not in the class- $\mathcal{C}$ but satisfies the $\partial \bar{\partial}$-lemma.

In a similar vein, one may ask whether there exist twistor spaces whose Frölicher spectral sequence degenerates at $E_{1}$ but on which the $\partial \bar{\partial}$-lemma does

10 The term semi-Kähler is used in [20] to mean balanced. 
not hold. As pointed out in the introduction ( $c f$. Proposition 1.8), the $\partial \bar{\partial}-$ lemma implies the Hodge symmetry. On the other hand, the Dolbeault cohomology of any twistor space $Z$ can be calculated in terms of the De Rham cohomology of the base four-manifold $M$ (see (4.1), (4.2), (4.4) taken from [16]). Hence the following are necessary conditions on the Betti numbers of $M$ for its twistor space $Z$ to satisfy the $\partial \bar{\partial}$-lemma:

$$
b_{1}(M)=b_{3}(M)=0 \quad \text { and } \quad b_{-}(M)=0,
$$

where $b_{-}(M):=\operatorname{dim}_{\mathbb{C}} H_{-}^{2}(M, \mathbb{C})$. Now the twistor spaces $\left(Z_{t}\right)_{t \in \Delta}$ in the EastwoodSinger family have $\left(M_{t}:=\mathbb{P}\left(E_{t}\right)\right)_{t \in \Delta}$ as respective underlying four-manifolds, where each $E_{t} \rightarrow \Sigma_{g}$ is a rank-two holomorphic vector bundle over a complex curve of genus $g \geq 2$. Thus each $M_{t}$ is a non-rational ruled surface having $b_{1}\left(M_{t}\right)=$ $2 g \geq 4$. In particular, $b_{1}\left(M_{t}\right) \neq 0$, showing that $M_{t}$ does not satisfy conditions (4.17). Hence no fibre in the Eastwood-Singer family of twistor spaces $\left(Z_{t}\right)_{t \in \Delta}$ can satisfy the $\partial \bar{\partial}$-lemma. Since $E_{1}\left(Z_{t}\right)=E_{\infty}\left(Z_{t}\right)$ for all $t \in \Delta \backslash\{0\}$, we obtain the following:

Observation 4.11. There exist twistor spaces whose Frölicher spectral sequence degenerates at $E_{1}$ but which do not satisfy the $\partial \bar{\partial}$-lemma. Any fibre $Z_{t}$ with $t \neq 0$ in the Eastwood-Singer family is such an example.

Another consequence of the Eastwood-Singer main theorem was that the Frölicher spectral sequence of the twistor space of any $K 3$ surface degenerates at $E_{1}$ ( $c f$. Corollary 5.2. in [16]). Now it is well-known that any $K 3$ surface $M$ satisfies

$$
b_{1}(M)=b_{3}(M)=0 \text { and } b_{-}(M)=19,
$$

meaning that the last of the conditions (4.17) is not satisfied by $K 3$ surfaces. Hence we have yet another class of examples illustrating Observation 4.11.

Observation 4.12. On the twistor space of any $K 3$ surface, the $\partial \bar{\partial}$-lemma does not hold although $E_{1}=E_{\infty}$ in the Frölicher spectral sequence.

It is thus natural to ask the following:

Question 4.13. Can one characterise the twistor spaces on which the $\partial \bar{\partial}-$ lemma holds? Is there a $\partial \bar{\partial}$-lemma analogue of the Eastwood-Singer description of the Frölicher degeneration property for twistor spaces?

In other words, it would be interesting to know whether there exists an equation similar to (4.5) or any other condition on $M$ that can be an indicator of the $\partial \bar{\partial}$-lemma property on $Z$.

We conclude by briefly mentioning another class of compact complex manifolds that usually provide a host of examples of various sorts: nilmanifolds. Recall that a connected, simply connected real Lie group $G$ possessing a discrete co-compact subgroup $\Gamma$ and a left-invariant complex structure $J$ defines a quotient compact complex manifold $X:=\Gamma \backslash G$ (inheriting its complex structure from $J$ by passing to the quotient) that is said to be a nilmanifold if the group $G$ is nilpotent. In the special case of a complex Lie group $G$, the quotient $X$ is a compact complex parallelisable nilmanifold (e.g. the Iwasawa manifold) of the class introduced 
by Wang in [48], but $G$ need not be a complex Lie group in general. Putting the following two pieces of information together:

- By [14], any compact complex manifold on which the $\partial \bar{\partial}$-lemma holds is formal.

- By [22], the only formal nilmanifolds are the complex tori (i.e. those defined by an Abelian Lie group $G$ ),

and since complex tori are clearly Kähler, we see that Kählerness and the $\partial \bar{\partial}$-lemma property are equivalent conditions on nilmanifolds. So we can ask whether one can construct a holomorphic family of nilmanifolds in which all the fibres, except one, are Kähler.

Added in proof. Conjectures 1.21 and 1.23 have been disproved in the paper Classification of complex structure on 6-dimensional nilpotent Lie algebras (arXiv e-print math.DG/1111.5873v4) by M. Ceballos, A. Otal, L. Ugarte and R. Villacampa.

\section{References}

[1] M. F. AtiYah, N. J. Hitchin and I. M. Singer, Self-duality in four-dimensional Riemannian geometry, Proc. Roy. Soc. London Ser. A 362 (1978), 425-461.

[2] L. AlesSANDrini and G. BASSANELli, Small deformations of a class of compact nonKähler manifolds, Proc. Amer. Math. Soc. 109 (1990), 1059-1062.

[3] L. Alessandrini and G. BAssanelli, Compact p-Kähler manifolds, Geom. Dedicata 38 (1991), 199-210.

[4] L. AleSSANDRINI and G. BASSANELli, Smooth proper modifications of compact Kähler manifolds, In: Proc. Internat. Workshop on Complex Analysis (Wuppertal 1990); Complex Analysis, Aspects of mathematics, Vol. E17, Vieweg, Braunschweig, 1991, 1-7.

[5] L. AlesSANDRINI and G. BASSANELli, Metric properties of manifolds bimeromorphic to compact Kähler spaces, J. Differential Geom. 37 (1993), 95-121.

[6] L. Alessandrini and G. BASSANelli, Modifications of compact balanced manifolds, C. R. Acad. Sci. Paris, Sér. I 320 (1995), 1517-1522.

[7] A. L. BESSE, "Einstein Manifolds", Springer, Berlin 1987.

[8] W. Barth, C. Peters and A. VAn De Ven, "Compact Complex Surfaces", SpringerVerlag, Berlin, 1984.

[9] N. BUCHDAHL, On compact Kähler surfaces, Ann. Inst. Fourier 49 (1999), 287-302.

[10] F. Campana, The class $\mathcal{C}$ is not stable by small deformations, Math. Ann. 290 (1991), $19-30$.

[11] F. CAMpana, On twistor spaces of the class $\mathcal{C}$, J. Differential Geom. 33 (1991), 541-549.

[12] E. CALABI and B. ECKMANN, A class of compact, complex manifolds which are not algebraic, Ann. of Math. 58 (1953), 494-500.

[13] L. A. Cordero, M. Fernandez, A. Gray and L. Ugarte, Compact nilmanifolds with nilpotent complex structures: Dolbeault cohomology, Trans. Amer. Math. Soc. 352 (2000), $5405-5433$.

[14] P. Deligne, Ph. Griffiths, J. Morgan and D. Sullivan, Real homotopy theory of Kähler manifolds, Invent. Math. 29 (1975), 245-274.

[15] J.-P. DemaILlY and M. PAUn, Numerical charaterization of the Kähler cone of a compact Kähler manifold, Ann. of Math. (2) 159 (2004), 1247-1274. 
[16] M. EASTwood and M. Singer, The Fröhlicher spectral sequence on a twistor space, J. Differential Geom. 38 (1993), 653-669.

[17] A. FuJIKI, Closedness of the Douady spaces of compact Kähler spaces, Publ. Res. Inst. Math. Sci., Kyoto Univ. 14 (1978), 1-52.

[18] P. Gauduchon Le théorème de l'excentricité nulle, C. R. Acad. Sci. Paris, Sér. A, 285 (1977), 387-390.

[19] P. GAUDUCHON, Fibrés hermitiens à endomorphisme de Ricci non négatif, Bull. Soc. Math. France 105 (1977), 113-140.

[20] P. Gauduchon, Structures de Weyl et théorèmes d'annulation sur une variété conforme autoduale, Ann. Scuola Norm. Sup. Pisa Cl. Sci. (4) 18 (1991), 563-629.

[21] R. HARTShorne, "Algebraic Geometry", Graduate Texts in Mathematics, No. 52, Springer-Verlag, New York-Heidelberg, 1977.

[22] K. Hasegawa, Minimal models of nilmanifolds, Proc. Amer. Math. Soc. 106 (1989), 6571.

[23] H. HironaKa, An example of a non-Kählerian complex-analytic deformation of Kählerian complex structures, Ann. of Math. (2) 75 (1962), 190-208.

[24] N. J. Hitchin, Kählerian twistor spaces, Proc. London Math. Soc. 43 (1981), 133-150.

[25] N. J. Hitchin, The self-duality equations on a Riemann surface, Proc. London Math. Soc. (3) 55 (1987), 59-126.

[26] H. Hopf, Zur topologie der komplexen mannigfaltigkeiten, In: "Studies and Essays Presented to R. Courant on his 60th Birthday”, Interscience Publishers Inc., New York, 1948 $167-185$.

[27] S. Ji, B. ShIFFMAn, Properties of compact complex manifolds carrying closed positive currents, J. Geom. Anal. 3 (1993), 37-61.

[28] K. KodAIRA, "Complex Manifolds and Deformations of Complex Structures", Grund. der Math. Wiss., Vol. 283, Springer, 1986.

[29] K. KodAIRA and D. C. SPENCER, On deformations of complex analytic structures III, stability theorems for complex structures, Ann. of Math. (2) 71 (1960), 43-76.

[30] M. KURANISHI, On the locally complete families of complex analytic structures, Ann. of Math. 75 (1962), 536-577.

[31] A. LAMARI, Courants kählériens et surfaces compactes, Ann. Inst. Fourier 49 (1999), 263285.

[32] C. Lebrun, On the topology of self-dual 4-manifolds, Proc. Amer. Math. Soc. 98 (1986), 637-640.

[33] C. LeBRUn, Explicit self-dual metrics on $\mathbb{C P}_{2} \sharp \ldots \sharp \mathbb{C P}_{2}$, J. Differential Geom. 34 (1991), 223-253.

[34] C. LebRUN and Y.-S. Poon, Twistors, Kähler manifolds, and bimeromorphic geometry, II, J. Amer. Math. Soc. 5, (1992), 317-325.

[35] M. L. Michelsohn, On the existence of special metrics in complex geometry, Acta Math. 143 (1983), 261-295.

[36] Y. MiYaokA, Kähler metrics on elliptic surfaces, Proc. Japan Acad. 50 (1974), 533-536.

[37] I. NAKAMURA, Complex parallelisable manifolds and their small deformations, J. Differential Geom. 10 (1975), 85-112.

[38] M. S. NARASIMHAN and S. RAMANAN, Moduli of vector bundles on a compact Riemann surface, Ann. of Math. 89 (1969), 14-51.

[39] M. S. NARASIMHAN and C. S. SESHADRI, Stable and unitary vector bundles on a compact Riemann surface, Ann. of Math. 82 (1965), 540-567.

[40] R. Penrose, Nonlinear gravitons and curved twistor theory, Gen. Relat. Gravitation 7 (1976), 31-52.

[41] D. PopovicI, Limits of projective manifolds under holomorphic deformations, arXiv e-print math.AG/0910.2032v1, and Deformations limits of projective manifolds: Hodge numbers and strongly Gauduchon metrics, Invent. Math. 194 (2013), 515-534. 
[42] D. Popovici, Limits of Moishezon manifolds under holomorphic deformations, arXiv eprint math.AG/1003.3605v1.

[43] D. PopovicI, Stability of strongly Gauduchon manifolds under modifications, J. Geom. Anal. 23 (2013), 653-659.

[44] M. SCHWEITZER, Autour de la cohomologie de Bott-Chern, arXiv e-print math. AG/0709.3528v1.

[45] Y.-T. SIU, Every K3 surface is Kähler, Invent. Math. 73 (1983), 139-150.

[46] H. Tsuji, Complex structures on $S^{3} \times S^{3}$, Tohoku Math. J. (2) 36 (1984), 351-376.

[47] J. VARouchas, Sur l'image d'une variété kählérienne compacte, In: LNM, Vol. 1188, Springer, 1986, 245-259.

[48] H. C. WANG, Complex parallisable manifolds, Proc. Amer. Math. Soc. 5 (1954), 771-776.

[49] C.-C. WU, "On the Geometry of Superstrings with Torsion", thesis, Department of Mathematics, Harvard University, Cambridge MA 02138, April 2006.

Institut de Mathématiques de Toulouse Université Paul Sabatier 118 route de Narbonne 31062 Toulouse Cedex 9, France popovici@math.ups-tlse.fr 